TRANSACTIONS OF THE

AMERICAN MATHEMATICAL SOCIETY

Volume 353, Number 4, Pages 1427-1453

S 0002-9947(00)02737-9

Article electronically published on November 29, 2000

\title{
THE INFORMATION ENCODED IN INITIAL IDEALS
}

\author{
GUNNAR FLØYSTAD AND MARK L. GREEN
}

\begin{abstract}
We consider homogeneous ideals $I$ and the initial ideal in $(I)$ for the revlex order. First we give a sequence of invariants computed from $I$ giving better and better "approximations" to the initial ideal and ending in an equivalent description.

Then we apply this to different settings in algebraic geometry to understand what information is encoded in the generic initial ideal of the ideal of a projective scheme.

We also consider the higher initial ideals as defined in a paper by Fløystad. In particular, we show that giving the generic higher initial ideal of a space curve is equivalent to giving the generic initial ideal of a linked curve.
\end{abstract}

\section{Introduction}

Consider the polynomial ring $S=k\left[x_{0}, \ldots, x_{r}\right]$ and let $I \subseteq S$ be a homogeneous ideal. If we have given a total order of the monomials in $S$, we may form the initial ideal in $(I)$. A very basic fact is that the ideal and the initial ideal have the same Hilbert function. But the initial ideal is a considerably more refined invariant than just the Hilbert function.

So one might ask what exactly is the extra information the initial ideal gives us. In this paper we address this question in the case that the monomial order is the reverse lexicographic order. We show, Theorem 2.9, that knowing the initial ideal in $(I)$ is equivalent to knowing the Hilbert functions of a set of modules computed from $S / I$ by standard operations from commutative algebra, like multiplying by elements of $S$, taking kernels and forming quotients. The point of this is that when $I$ defines a variety $X \subseteq \mathbf{P}^{r}$, this enables us to relate the initial ideal to algebraic geometric properties of $X$. Thus we show how many properties of $X$, mainly of a cohomological nature, may be computed from the initial ideal in $(I)$ for the reverse lexicographic order.

In section 3 we apply this to the case of a space curve $C \subseteq \mathbf{P}^{3}$. We show, Theorem 3.5 , that the generic initial ideal of $I_{C}$ is equivalent to i) the Hilbert functions of certain submodules computed from the Hartshorne-Rao module $\bigoplus_{n \in \mathbf{Z}} H^{1} \mathcal{I}_{C}(n)$, and ii) the numerical character of $C$. They are equivalent in the sense that these two sets of data may be computed from each other.

In section 4 we apply this to the case of a zero-dimensional scheme $X \subseteq \mathbf{P}^{3}$. We show that the generic initial ideal of $I_{X}$ is equivalent to the Hilbert functions of certain submodules of the module $\bigoplus_{n \in \mathbf{Z}} H^{1} \mathcal{I}_{X}(n)$.

Received by the editors June 5, 1999

2000 Mathematics Subject Classification. Primary 13P10; Secondary 14H50.

(C)2000 American Mathematical Society 
In section 5 we consider the intersection of a curve $C$ and a surface $F$ in $\mathbf{P}^{3}$ of sufficiently high degree. Let $I=I_{C}+I_{F}$. We show that all cohomological dimensions $\operatorname{dim}_{k} H^{i} \mathcal{I}_{C}(n)$ of the curve may be computed from the generic initial ideal of $I$.

Now for a space curve $C \subseteq \mathbf{P}^{3}$ there is, beside the generic initial ideal, also the generic higher initial ideal [5], denoted $\operatorname{gin}_{1}\left(I_{C}\right)$. Having achieved an understanding of the generic initial ideal of $I_{C}$ it is natural to try to also get an understanding of the generic higher initial ideal. In order to do so we have to consider the formation of initial ideals in a more general setting.

We have homogeneous $S$-modules $U$ and $R$ where in addition $R$ has a $\mathbf{Z}^{r+1}$ grading compatible with the natural $\mathbf{Z}^{r+1}$-grading on $S$. There will be a map

$$
\text { in }: U \longrightarrow R
$$

with properties making it natural to call it an initial map. In section 6 we isolate these properties, abstract the situation and develop some simple theory for this which generalizes the theory we have for initial ideals.

In the last section we apply the above to the process involved in forming the generic higher initial ideal of $I_{C}$. The result we get is in complete analogy to the result for the generic initial ideal of $I_{C}$. Namely, we show that the generic higher initial ideal of $I_{C}$ is equivalent to i) the Hilbert functions of certain quotient modules of the Hartshorne-Rao module $\bigoplus_{n \in \mathbf{Z}} H^{1} \mathcal{I}_{C}(n)$, and ii) the numerical character of $C$.

As a consequence we show that if the curve $C$ is linked to a curve $X$, then knowing the generic higher initial ideal of $I_{C}$ is equivalent to knowing the generic initial ideal of $I_{X}$.

Terminology. If we have two sets of data $A$ and $B$ such that $B$ may be computed from $A$ and $A$ may be computed form $B$, then we say that $A$ and $B$ are computationally equivalent. When we give such a statement in a proposition, it will be clear from the proof or by reference in the proof to earlier propositions how these computations may be done.

Note. Theorems 3.5, 7.10 and 7.11 concerning space curves have independently and, we believe, prior to us been shown by R. Liebling in his thesis [10]. He has however his own definition of the higher initial ideal rather than the original [5], which we use. He works specifically in the context of space curves, where he probes deeper than we do.

\section{Preliminiaries}

1.1. Let $S=k\left[x_{0}, \ldots, x_{r}\right]$ be the polynomial ring with char $k=0$. We denote by $S_{d}$ the direct summand in $S$ of degree $d$. If $K=\left(k_{0}, \ldots, k_{r}\right)$ we use the notation

$$
x^{K}=x_{0}^{k_{0}} \cdots x_{r}^{k_{r}}
$$

for monomials. Its degree is $|K|=\sum_{j=0}^{r} k_{j}$.

1.2. The initial ideal. Suppose we have a total order on the monomials. For $f=\sum a_{K} x^{K}$ a homogeneous polynomial, let the initial monomial be

$$
\operatorname{in}(f)=\max \left\{x^{K} \mid a_{K} \neq 0\right\}
$$


If $I \subseteq S$ is a homogeneous ideal, the initial ideal is the ideal generated by all the initial monomials of homogeneous polynomials in $I$ :

$$
\operatorname{in}(I)=(\{\operatorname{in}(f) \mid f \in I\}) .
$$

1.3. The Hilbert function. For a homogeneous module $M$ over $S$ let $\operatorname{Hilb}(M)$ : $\mathbf{Z} \rightarrow \mathbf{N}_{\mathbf{0}}$ be its Hilbert function, i.e. $\operatorname{Hilb}(M)(n)=\operatorname{dim}_{k} M_{n}$. It is a well known fact that the ideal $I$ and its initial ideal in $(I)$ have the same Hilbert function.

1.4. The function $p$. Define the function

$$
p: \mathbf{N}_{\mathbf{0}}{ }^{r} \longrightarrow \mathbf{N}_{\mathbf{0}} \cup\{\infty\}
$$

by

$$
p\left(k_{0}, \ldots, k_{r-1}\right)=\min \left\{k_{r} \mid x_{0}^{k_{0}} \cdots x_{r-1}^{k_{r-1}} x_{r}^{k_{r}} \in \operatorname{in}(I)\right\} .
$$

We see that $\operatorname{in}(I)$ is determined by the function $p$. So this is a way to represent the initial ideal.

1.5. Generic initial ideal and Borel fixedness. If $g \in G L\left(S_{1}\right)$ is a change of coordinates, then we get $g . I \subseteq S$ and its initial ideal in $(g . I)$. There is an open subset $U \subseteq G L\left(S_{1}\right)$ such that in $(g . I)$ is the same for all $g \in U$, see [4] 15.9. This initial ideal is called the generic initial ideal of $I$. It is denoted as gin $(I)$.

A basic fact (see [4, Theorem 15.20, or originally 7]) is that $\operatorname{gin}(I)$ is Borel-fixed. That is, if $\mathbf{x}^{K}$ is a monomial in $\operatorname{gin}(I)$ and $x_{j}$ divides $\mathbf{x}^{K}$, then $x_{i} \mathbf{x}^{K} / x_{j} \in \operatorname{gin}(I)$ for all $0 \leq i<j$. This means that for $K \in \mathbf{N}_{\mathbf{0}}{ }^{r}$ and $e_{i}=(0, \ldots, 1, \ldots, 0)$ is the $i$ 'th coordinate vector, then

1. $p\left(K+e_{i}\right) \leq p\left(K+e_{j}\right)$ for $0 \leq i<j \leq r-1$,

2. $p\left(K+e_{r-1}\right) \leq p(K)-1$ if $p(K)>0$.

1.6. Revlex order. The monomial order we consider in this paper will be the reverse lexicographic order. Then

$$
x^{K}>x^{J}
$$

if $|K|>|J|$ or if $k_{r}<j_{r}$, where $r$ is maximal such that $k_{r} \neq j_{r}$. Intuitively $x^{J}$ is dragged down by having a "large weight in the rear".

1.7. Identities for revlex order. In the case of the revlex order there is a commutative diagram (4], Proposition 15.12 a)

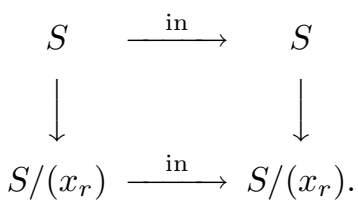

We denote by $I_{\mid x_{r}=0}$ the image of the composition

$$
I \longrightarrow S \longrightarrow S /\left(x_{r}\right) \text {. }
$$

Then we see that this gives the identity

$$
\operatorname{in}\left(I_{\mid x_{r}=0}\right)=\operatorname{in}(I)_{\mid x_{r}=0} .
$$

Also we have the identity

$$
\operatorname{in}\left(I: x_{r}\right)=\operatorname{in}(I): x_{r}
$$

by [4], Proposition 15.12 b. 
1.8. Saturation. The saturation $I^{\text {sat }}$ of $I$ is by definition

$$
\bigcup_{k \geq 0}\left(I:\left(x_{0}, \ldots, x_{r}\right)^{k}\right) \text {. }
$$

If $h$ is a general linear form, this is by [8], Proposition 2.19, equal to $\left(I: h^{k}\right)$ for $k \gg 0$. In particular, if $I$ is in general coordinates, then the saturation is $\left(I: x_{r}^{k}\right)$ for $k \gg 0$.

1.9. If $\mathcal{F}$ is a sheaf on the projective space $\mathbf{P}^{r}$ over $k$, we use the notation $H_{*}^{i} \mathcal{F}=$ $\bigoplus_{n \in \mathbf{Z}} H^{i} \mathcal{F}(n)$. It is a graded module over $S=k\left[x_{0}, \ldots, x_{r}\right]$. We also use the notation $h^{i} \mathcal{F}(n)=\operatorname{dim}_{k} H^{i} \mathcal{F}(n)$. Note that if $I \subseteq S$ defines a scheme $X$, and if $\mathcal{I}$ is the ideal sheaf associated to $I$ then

$$
H_{*}^{0} \mathcal{I}=I_{X}=I^{s a t},
$$

the saturated homogeneous ideal associated to $X$.

\section{INITIAL IDEALS}

In this section we consider a homogeneous ideal $I \subseteq S$. We may form its initial ideal in $(I)$. Here we will give some results telling us what kind of information the initial ideal contains.

As we said in 1.2, the ideal $I$ and the initial ideal in $(I)$ have the same Hilbert function. But the initial ideal is a more refined discrete invariant than the Hilbert function. So what extra information is contained in the initial ideal? We shall give an answer to this in terms of a sequence of data. Each piece of data will be more refined than the previous, and in the end we will have a set of data which is equivalent to the initial ideal.

2.1. We have ideals $\left(I: x_{r}^{j}\right)$ for $j \geq 0$. Since $S$ is Noetherian, the ascending chain of ideals

$$
I \subseteq \cdots \subseteq\left(I: x_{r}^{j-1}\right) \subseteq\left(I: x_{r}^{j}\right) \subseteq \cdots
$$

stabilizes in an ideal which we denote $\left(I: x_{r}^{\infty}\right)$. For $j \geq 0$ or $j=\infty$ let

$$
F^{j}(I)=\left(I: x_{r}^{j}\right) / I .
$$

Note that $F^{j}(I)$ is the kernel of the map

$$
S / I \stackrel{\cdot x_{r}^{j}}{\longrightarrow}(S / I)(j) .
$$

Furthermore, for $j \geq 0$ or $j=\infty$ let

$$
I^{j}=\operatorname{im}\left(\left(I: x_{r}^{j}\right) \rightarrow S \rightarrow S /\left(x_{r}\right)\right) .
$$

This is of course not the $j$ 'th power of the ideal. No confusion should result from this in this paper.

Lemma 2.2. The $I^{j}$ and $F^{j}(I)$ are related as follows.

a. There is an exact sequence

$$
0 \longrightarrow F^{j-1}(I) \longrightarrow F^{j}(I) \longrightarrow F^{j}(I) / F^{j-1}(I) \longrightarrow 0 .
$$

$b$. There is an exact sequence

$$
\begin{aligned}
0 \longrightarrow F^{j+1}(I) / F^{j}(I)(-1) & \stackrel{\cdot x_{r}}{\longrightarrow} F^{j}(I) / F^{j-1}(I) \\
& \longrightarrow F^{j}(I) /\left(F^{j-1}(I)+x_{r} \cdot F^{j+1}(I)\right) \longrightarrow 0 .
\end{aligned}
$$


c. There is an isomorphism

$$
I^{j} / I^{j-1}=F^{j}(I) /\left(F^{j-1}(I)+x_{r} \cdot F^{j+1}(I)\right) .
$$

Proof. a. The sequence (2.2) is clear.

b. That the sequence (2.3) is right exact is also clear. Suppose then that $\bar{v} \in$ $F^{j+1}(I) \subseteq S / I$ is such that $x_{r} \cdot \bar{v} \in F^{j-1}(I)$. Then $x_{r}^{j} \cdot \bar{v}=\overline{0}$ in $S / I$ by the description of $F^{j-1}(I)$ in (2.1). Hence $\bar{v} \in F^{j}(I)$. Thus the first map in (2.3) is injective.

c. There is clearly a surjection

$$
F^{j}(I)=\left(I: x_{r}^{j}\right) / I \stackrel{p}{\longrightarrow}\left(I: x_{r}^{j}\right) /\left(\left(x_{r}\right)+I\right) .
$$

If a non-zero $\bar{u} \in F^{j}(I)$ is such that $p(\bar{u})=\overline{0}$, we may assume that $u=x_{r} \cdot v$ in $S$. Then $x_{r}^{j+1} \cdot v=x_{r}^{j} \cdot u \in I$, so $v \in\left(I: x_{r}^{j+1}\right)$. Hence $\operatorname{ker}(p)=x_{r} \cdot F^{j+1}(I)$ and

$$
F^{j}(I) /\left(x_{r} \cdot F^{j+1}(I)\right)=\left(I: x_{r}^{j}\right) /\left(\left(x_{r}\right)+I\right) .
$$

This gives

$$
F^{j}(I) /\left(F^{j-1}(I)+x_{r} \cdot F^{j+1}(I)\right)=\left(I: x_{r}^{j}\right) /\left(\left(x_{r}\right)+\left(I: x_{r}^{j-1}\right)\right)=I^{j} / I^{j-1} .
$$

We now have, almost immediately,

Algorithm 2.3. The Hilbert functions of the modules in the filtration

$$
0=F^{0}(I) \subseteq F^{1}(I) \subseteq \cdots \subseteq F^{\infty}(I)
$$

are computationally equivalent to the Hilbert functions of the modules $I^{j} / I^{j-1}$. In fact, let

$$
f^{j}, \quad \bar{f}^{j}, \quad \tilde{f}^{j}
$$

be the Hilbert functions of

$$
F^{j}(I), \quad F^{j}(I) / F^{j-1}(I), \quad I^{j} / I^{j-1}
$$

respectively. Then they are related by

$a$.

$$
\begin{aligned}
& \tilde{f}^{j}(n)=\bar{f}^{j}(n)-\bar{f}^{j+1}(n-1), \\
& \bar{f}^{j}(n)=f^{j}(n)-f^{j-1}(n),
\end{aligned}
$$

$b$.

$$
\begin{aligned}
& \bar{f}^{j}(n)=\tilde{f}^{j}(n)+\tilde{f}^{j+1}(n-1)+\cdots+\tilde{f}^{j+r}(n-r)+\cdots, \\
& f^{j}(n)=\bar{f}^{j}(n)+\bar{f}^{j-1}(n)+\cdots+\bar{f}^{1}(n) .
\end{aligned}
$$

Proof. a. This is clear by Lemma 2.2

b. This follows from a.

Corollary 2.4. The Hilbert function of I may be computed from the Hilbert functions of the $I^{j}$ for $j \geq 0$. 
Proof. By the exact sequence

$$
0 \longrightarrow F^{1}(I) \longrightarrow S / I \stackrel{\cdot x_{r}}{\longrightarrow}(S / I)(1) \longrightarrow\left(k\left[x_{0}, \ldots, x_{r-1}\right] / I^{0}\right)(1) \longrightarrow 0
$$

we see that $\operatorname{Hilb}(I)$ may be computed from $\operatorname{Hilb}\left(F^{1}(I)\right)$ and $\operatorname{Hilb}\left(I^{0}\right)$. But $\operatorname{Hilb}\left(F^{1}(I)\right)$ may be computed from $\operatorname{Hilb}\left(I^{j} / I^{j-1}\right)$ for $j \geq 1$ according to Algorithm 2.3.

Remark 2.5. The converse is not true in general. Thus the Hilbert functions of the $I^{j}$ for $j \geq 0$ constitutes a more refined invariant of $I$ than the Hilbert function of $I$.

Lemma 2.6. Let I be a monomial ideal. Then I is computationally equivalent to $I^{j}$ for all $j \geq 0$. In fact,

$$
x_{0}^{i_{0}} \cdots x_{r-1}^{i_{r-1}} \in I^{j} \Leftrightarrow x_{0}^{i_{0}} \cdots x_{r-1}^{i_{r-1}} x_{r}^{j} \in I .
$$

Proof. This is obvious.

Lemma 2.7. Let $I \subseteq S$ be a homogeneous ideal. Then

$$
i n\left(I^{j}\right)=i n(I)^{j}
$$

Proof. By 1.7 there is a commutative diagram

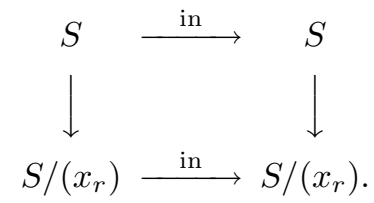

Apply this to $\left(I: x_{r}^{j}\right) \subseteq S$ and use (1.3) that in $\left(I: x_{r}^{j}\right)=\operatorname{in}(I): x_{r}^{j}$.

We now get the following.

Proposition 2.8. Consider the following data:

$$
\begin{aligned}
& \text { i. } i n(I), \quad \text { ii. } i n\left(I^{j}\right) \text { for } j \geq 0 \text {. } \\
& \text { iii. } \operatorname{Hilb}\left(I^{j}\right) \text { for } j \geq 0, \quad i v \text {. } \operatorname{Hilb}(I) \text {. }
\end{aligned}
$$

a. Data i. and ii. are computationally equivalent. Data iii. may be computed from data ii. and data iv. may be computed from data iii.

$b$. If I is saturated and in general coordinates, then $i$. and $i$. are computationally equivalent to in $\left(I^{0}\right)$.

Proof. a. That data $i$. and $i i$. are computationally equivalent follows from Lemma 2.6 and Lemma 2.7. That data iii. may be computed from data ii. follows from 1.3 , and that data $i v$. may be computed from data iii. follows from Corollary 2.4

b. If $I$ is saturated and in general coordinates, then $\left(I: x_{r}^{j}\right)=I$ according to 1.8. Thus $I^{j}=I^{0}$ for all $j \geq 0$.

Remark 2.9. By the above Proposition 2.8 a. and Remark 2.5 we see that the $\operatorname{Hilb}\left(I^{j}\right)$ for $j \geq 0$ can be considered a better approximation to in $(I)$ than $H i l b(I)$.

Proposition 2.8 says that we can compute the $\operatorname{Hilb}\left(I^{j}\right)$ for $j \geq 0$ from in $(I)$. The following proposition gives us an explicit way of doing this by enabling us to compute $\operatorname{Hilb}\left(I^{\infty}\right)$ and $\operatorname{Hilb}\left(I^{j} / I^{j-1}\right)$ for $j \geq 1$. 
Proposition 2.10. Let $p$ be the function from 1.4 associated to in $(I)$.

a. Let $K \in \mathbf{N}_{\mathbf{0}}{ }^{r}$. Then $x^{K} \in \operatorname{in}\left(I^{\infty}\right) \Leftrightarrow p(K)<\infty$.

b. The Hilbert function $\tilde{f}^{j}$ of $I^{j} / I^{j-1}$ is given by

$$
\tilde{f}^{j}(n)=\#\left\{K \in \mathbf{N}_{\mathbf{0}}{ }^{r}|| K \mid=n, p(K)=j\right\} .
$$

Proof. a. Since $\operatorname{in}\left(I^{\infty}\right)=\left(\operatorname{in}(I): x_{r}^{\infty}\right) /\left(x_{r}\right)$, we see that for $K \in \mathbf{N}_{\mathbf{0}}{ }^{r}$ then

$$
\begin{aligned}
x^{K} \in \operatorname{in}\left(I^{\infty}\right) & \Leftrightarrow x^{K} \cdot x_{r}^{j} \in \operatorname{in}(I) \text { for } j \gg 0 \\
& \Leftrightarrow p(K)<\infty .
\end{aligned}
$$

b. From Lemma 2.6 we see that

$$
\overline{x^{K}} \in \operatorname{in}(I)^{j} / \operatorname{in}(I)^{j-1} \Leftrightarrow p(K)=j .
$$

Since $I^{j} / I^{j-1}$ and $\operatorname{in}(I)^{j} / \operatorname{in}(I)^{j-1}$ have the same Hilbert functions by Lemma 2.7 it follows that

$$
\tilde{f}^{j}(n)=\#\left\{K \in \mathbf{N}_{\mathbf{0}}{ }^{r}|| K \mid=n, p(K)=j\right\} .
$$

Successive approximations. The view we now pursue is the one given in Remark 2.9. namely that the $\operatorname{Hilb}\left(I^{j}\right)$ for $j \geq 0$ are a better approximation to in $(I)$ than $\operatorname{Hilb}(I)$ is. We now define inductively

$$
F^{j_{1}, \ldots, j_{m}, j}(I)=F^{j}\left(I^{j_{1}, \ldots, j_{m}}\right) .
$$

Alternatively we have

$$
F^{j_{1}, \ldots, j_{m}, j}(I)=\left(I^{j_{1}, \ldots, j_{m}}: x_{r-m}^{j}\right) / I^{j_{1}, \ldots, j_{m}} .
$$

Correspondingly we have

$$
\begin{aligned}
I^{j_{1}, \ldots, j_{m}, j}=\operatorname{im}\left(\left(I^{j_{1}, \ldots, j_{m}}: x_{r-m}^{j}\right)\right. & \longrightarrow k\left[x_{0}, \ldots, x_{r-m}\right] \\
& \left.\longrightarrow k\left[x_{0}, \ldots, x_{r-m}\right] /\left(x_{r-m}\right)\right) .
\end{aligned}
$$

Proposition 2.11. Suppose $b \geq a$. Then the $\operatorname{Hilb}\left(I^{\mathbf{j}}\right)$ for $\mathbf{j} \in \mathbf{N}_{\mathbf{0}}{ }^{a}$ may be computed from the Hilb( $\left.I^{\mathbf{k}}\right)$ for $\mathbf{k} \in \mathbf{N}_{0}{ }^{b}$.

Proof. This follows by repeated use of Corollary 2.4.

Theorem 2.12. Given $a \geq 0$. Consider the following data.

$$
\text { i. in }(I), \quad \text { ii. } \operatorname{in}\left(I^{\mathbf{j}}\right) \text { for } \mathbf{j} \in \mathbf{N}_{0}{ }^{a}, \quad \text { iii. } \operatorname{Hilb}\left(I^{\mathbf{j}}\right) \text { for } \mathbf{j} \in \mathbf{N}_{0}{ }^{a} \text {. }
$$

a. Data i. and ii. are computationally equivalent. Data iii. may be computed from data ii.

b. If $a=r$ then data iii. is computationally equivalent to data ii.

c. If $a=r-1$ and $I$ is in general coordinates so that in $(I)$ is Borel fixed, then data iii. is computationally equivalent to data ii.

Proof. a. That data $i$. and data $i i$. are computationally equivalent follows by repeated use of Proposition $2.8 \mathrm{a}$. That data iii. can be computed from data $i$. follows from 1.3.

b. If $a=r$ then $I^{\mathbf{j}} \subseteq k\left[x_{0}\right]$, but then in $\left(I^{\mathbf{j}}\right)$ is clearly computationally equivalent to $\operatorname{Hilb}\left(I^{\mathbf{j}}\right)$. 
c. If $a=r-1$ then $I^{\mathbf{j}} \subseteq k\left[x_{0}, x_{1}\right]$. If $\operatorname{in}(I)$ is Borel fixed, so is $\operatorname{in}(I)^{\mathbf{j}}=\operatorname{in}\left(I^{\mathbf{j}}\right)$. But a Borel fixed ideal $\operatorname{in}\left(I^{\mathbf{j}}\right) \subseteq k\left[x_{0}, x_{1}\right]$ is determined by its Hilbert function $\operatorname{Hilb}\left(I^{\mathbf{j}}\right)$, so c. follows.

Remark 2.13. By Proposition2.11 and the above Theorem 2.12 we see that $\operatorname{Hilb}\left(I^{\mathrm{j}}\right)$ for $\mathbf{j} \in \mathbf{N}_{0}{ }^{a}$ constitute a finer and finer set of invariants of $I$ as $a$ increases, and when $a=r$ we get a set of invariants which is computationally equivalent to in $(I)$. We therefore get successively better approximations to in $(I)$, which in the end gives an equivalent description of in $(I)$.

\section{Applications to space curves I}

3.1. Consider a space curve $C \subseteq \mathbf{P}^{3}$. Let $I_{C} \subseteq S=k\left[x_{0}, x_{1}, x_{2}, x_{3}\right]$ be its homogeneous ideal. We would like to understand what information is contained in the generic initial ideal $\operatorname{gin}\left(I_{C}\right)$. If we assume $I_{C}$ to be given in general coordinates, then this is just the initial ideal $\operatorname{in}\left(I_{C}\right)$.

Proposition 3.2. Let $J=I_{C}{ }^{0}$.

a. The initial ideals in $\left(I_{C}\right)$ and in $(J)$ are computationally equivalent.

b. in $(J)$ is computationally equivalent to:

i. The Hilbert function of $J^{\infty}$.

ii. The Hilbert functions of the modules in the filtration

$$
0=F^{0}(J) \subseteq F^{1}(J) \subseteq \cdots \subseteq F^{\infty}(J) .
$$

Proof. a. Since $I_{C}$ is saturated and in general coordinates, this is Proposition 2.8 b.

b. Since $J \subseteq k\left[x_{0}, x_{1}, x_{2}\right]$, by Theorem [2.12 c. in $(J)$ is computationally equivalent to $\operatorname{Hilb}\left(J^{j}\right)$ for all $j \geq 0$. This is again computationally equivalent to $\operatorname{Hilb}\left(J^{\infty}\right)$ and $\operatorname{Hilb}\left(J^{j} / J^{j-1}\right)$ for $j \geq 1$, and the latter is by Algorithm 2.3 computationally equivalent to $\operatorname{Hilb}\left(F^{j}(J)\right)$ for all $j \geq 0$.

Remark 3.3. Proposition 2.10 and Algorithm 2.3 show how in $(J)$ and data b.i. and b.ii. in the above Proposition 3.2 may be computed from each other.

We would like to find more algebraic geometric descriptions of the data in b.i. and $b$.ii of the above Proposition 3.2. First we recall two basic invariants from space curves.

The numerical character. Let $\Gamma=C \cap\left\{x_{3}=0\right\}$, which is a general plane section of the curve $C$. The saturated homogeneous ideal $I_{\Gamma}$ is $J^{\text {sat }}=\left(J: x_{2}^{\infty}\right)$ by 1.8 .

Lemma 3.4. a. The data i. in $\left(I_{\Gamma}\right)$, ii. in $\left(I_{\Gamma}^{0}\right)$, and iii. $H i l b\left(I_{\Gamma}{ }^{0}\right)$ are all computationally equivalent.

$$
\text { b. } I_{\Gamma}^{0}=J^{\infty} \text {. }
$$

Proof. a. Since $I_{\Gamma}$ is saturated, Proposition 2.8 b. gives that $\operatorname{in}\left(I_{\Gamma}\right)$ is computationally equivalent to in $\left(I_{\Gamma}{ }^{0}\right)$, which again by Theorem 2.12 $\mathrm{c}$. is computationally equivalent to $\operatorname{Hilb}\left(I_{\Gamma^{0}}\right)$.

b. follows if we consider the image of $I_{\Gamma}=\left(J: x_{2}^{\infty}\right)$ by the map

$$
k\left[x_{0}, x_{1}, x_{2}\right] \rightarrow k\left[x_{0}, x_{1}, x_{2}\right] /\left(x_{2}\right) .
$$


Remark 3.5. If $I_{\Gamma}^{0}=\left(x_{0}^{h}, x_{0}^{h-1} x_{1}^{\lambda_{h-1}}, \ldots, x_{1}^{\lambda_{0}}\right)$, then the numerical character of $C$ is the sequence of data $\left(n_{0}, \ldots, n_{h-1}\right)$, where $n_{j}=\lambda_{j}+j$ for $j=0, \ldots, h-1$ (see 8], p.55/56, or 9]). Thus we see that $\operatorname{Hilb}\left(J^{\infty}\right)$ is computationally equivalent to the numerical character of the curve $C$.

The Hartshorne-Rao module. This is the module

$$
H_{*}^{1} \mathcal{I}_{C}=\bigoplus_{n \in \mathbf{Z}} H^{1} \mathcal{I}_{C}(n) \text {. }
$$

When $C$ is a (scheme-theoretic) reduced union of integral curves, this is a finite length graded module over $S$. It plays a fundamental role in the study of space curves.

\section{Lemma 3.6.}

$$
F^{\infty}(J)=\operatorname{ker}\left(H_{*}^{1} \mathcal{I}_{C}(-1) \stackrel{\cdot x_{3}}{\longrightarrow} H_{*}^{1} \mathcal{I}_{C}\right)
$$

Proof. There is a short exact sequence of ideal sheaves

$$
0 \longrightarrow \mathcal{I}_{C}(-1) \stackrel{\cdot x_{3}}{\longrightarrow} \mathcal{I}_{C} \longrightarrow \mathcal{I}_{\Gamma} \longrightarrow 0 .
$$

Taking the long exact cohomology sequence of this short exact sequence, we get

$$
\begin{aligned}
0 & \longrightarrow I_{C}(-1) \stackrel{\cdot x_{3}}{\longrightarrow} I_{C} \stackrel{\alpha}{\longrightarrow} I_{\Gamma} \\
& \longrightarrow H_{*}^{1} \mathcal{I}_{C}(-1) \stackrel{\cdot x_{3}}{\longrightarrow} H_{*}^{1} \mathcal{I}_{C} \longrightarrow H_{*}^{1} \mathcal{I}_{\Gamma} .
\end{aligned}
$$

We see that $J=\alpha\left(I_{C}\right)$, and so

$$
F^{\infty}(J)=J^{\text {sat }} / J=I_{\Gamma} / \alpha\left(I_{\Gamma}\right)=\operatorname{ker}\left(H_{*}^{1} \mathcal{I}_{C}(-1) \stackrel{\cdot x_{3}}{\longrightarrow} H_{*}^{1} \mathcal{I}_{C}\right)
$$

We now have the following.

Theorem 3.7. Let $C \subseteq \mathbf{P}^{3}$ be a space curve. Let $h$ and $h^{\prime}$ be general linear forms. Let

$$
F^{\infty}(1)=\operatorname{ker}\left(H_{*}^{1} \mathcal{I}_{C} \stackrel{\cdot h}{\longrightarrow} H_{*}^{1} \mathcal{I}_{C}(1)\right)
$$

and let $F^{j}=\operatorname{ker}\left(F^{\infty} \stackrel{\cdot\left(h^{\prime}\right)^{j}}{\longrightarrow} F^{\infty}(j)\right)$. Then in $\left(I_{C}\right)$ is computationally equivalent to:

i. The numerical character of $C$.

ii. The Hilbert functions of the modules in the filtration

$$
0=F^{0} \subseteq F^{1} \subseteq \cdots \subseteq F^{\infty} .
$$

Proof. Since $I_{C}$ is in general coordinates, $x_{2}$ and $x_{3}$ can be considered as general linear forms $h^{\prime}$ and $h$. The theorem then follows by Proposition 3.2, Remark 3.5. and Lemma 3.6, since $F^{j}(J)=\operatorname{ker}\left(F^{\infty}(J) \stackrel{\cdot x_{2}}{\longrightarrow} F^{\infty}(J)(1)\right)$.

Example 3.8. The following example was brought to our attention by Chris von Bothmer. Consider a smooth rational quintic curve $C$. What may the initial ideal look like? 
General curve. Taking a general example and computing on Macaulay, one finds the initial ideal to be the following (we represent it in Figure 1 by means of the numbers $p\left(k_{0}, k_{1}\right)$ associated to the initial ideal of $\left.J=I_{C}{ }^{0}\right)$ :

Fig. 1
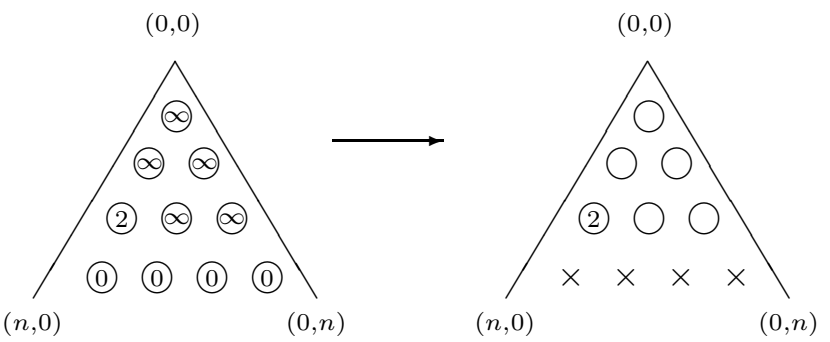

Numerical character. Since $J^{\infty}=I_{\Gamma}{ }^{0}$ we see by Proposition 2.10] that in $\left(I_{\Gamma}{ }^{0}\right) \operatorname{can}$ be represented by the diagram in Figure 2 . Here we have $\times$ in position $\left(k_{0}, k_{1}\right)$ if $x_{0}^{k_{0}} x_{1}^{k_{1}} \in \operatorname{in}\left(I_{\Gamma}^{0}\right)$ and $\bigcirc$ in position $\left(k_{0}, k_{1}\right)$ if $x_{0}^{k_{0}} x_{1}^{k_{1}} \notin \operatorname{in}\left(I_{\Gamma}^{0}\right)$. So we see that the invariants are $\left(\lambda_{0}, \lambda_{1}\right)=(3,2)$ and the numerical character is $\left(n_{0}, n_{1}\right)=(3,3)$.

Fig. 2

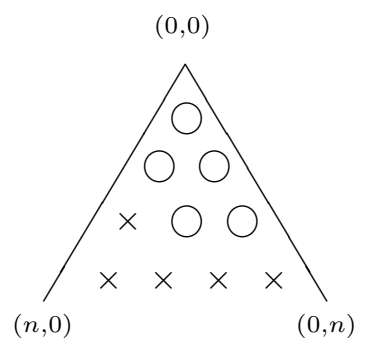

Other cases. Now there are three other candidates for the initial ideal of a rational quintic curve when you take into consideration that the curve has connected numerical character (see [8], Corollary 4.8) and that the generic initial ideal is Borel-fixed:
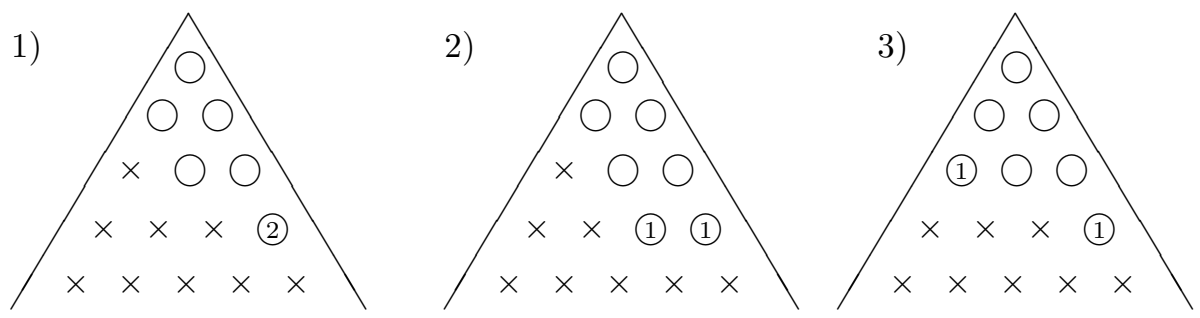

Case 1) may be excluded as in $(I)$ by observing that in this case $C$ must lie on a quadric and a cubic surface. Linking $C$ with these will give a line, so $C$ must be projectively Cohen-Macaulay and have $H_{*}^{1} \mathcal{I}_{C}=0$. By sequence (3.1) this means 
that $J=J^{\text {sat }}$, so $J^{j}=J^{0}$ for all $j \geq 0$. Then $\operatorname{in}\left(J^{j}\right) / \operatorname{in}\left(J^{j-1}\right)=0$ for all $j \geq 1$. This means by Proposition 2.10 that for no $\left(k_{0}, k_{1}\right)$ do we have $p\left(k_{0}, k_{1}\right)=j$ when $1 \leq j<\infty$. Thus 1 ) cannot be the diagram of $C$.

Case 2) would have to be a rational curve on a quadric surface. Since a curve of type $(4,1)$ on a smooth quadric surface is such a curve, its initial ideal must be that of 2) since 1) is excluded.

Case 3) poses more problems. It has exactly the same Hilbert function as in the first computed example. However, suppose the diagram represented in $(J)$. One sees by Proposition 2.10 that the Hilbert function $\tilde{f}^{j}=0$ for $j \neq 1$. For $j=1$ one finds that $\tilde{f}^{1}(2)=1, \tilde{f}^{1}(3)=1$ and $\tilde{f}^{1}(n)=0$ otherwise. By Algorithm 2.3 we deduce the following tables:

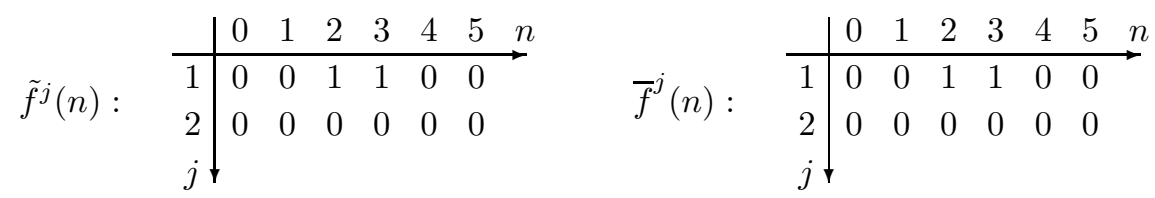

We now get the following table for the Hilbert functions of the filtration of $F^{\infty}$, and to the right we have the corresponding table for the filtration of $F^{\infty}(1)$ :

\begin{tabular}{cc|cccccccc|ccccccc} 
& & 0 & 1 & 2 & 3 & 4 & 5 & $n$ & & 0 & 1 & 2 & 3 & 4 & 5 & $n$ \\
$f^{j}(n):$ & 1 & 0 & 0 & 1 & 1 & 0 & 0 & 1 & 0 & 1 & 1 & 0 & 0 & 0 & \\
& 2 & 0 & 0 & 1 & 1 & 0 & 0 & 2 & 0 & 1 & 1 & 0 & 0 & 0
\end{tabular}

From the Hilbert function of $I_{\Gamma}$ one may compute $h^{1} \mathcal{I}_{\Gamma}(n)$. Doing so, one finds that $h^{1} \mathcal{I}_{\Gamma}(n)=0$ for $n \geq 2$.

We now wish to find $h^{1} \mathcal{I}_{C}(n)$. For this we repeatedly use Lemma 3.6 and sequence (3.1). But first note that since the curve is smooth we have $h^{1} \mathcal{I}_{C}(n)=0$ for $n \leq 0$.

We claim that $h^{1} \mathcal{I}_{C}(n)=0$ for $n \geq 3$. This is because $h^{1} \mathcal{I}_{C}(n-1)=h^{1} \mathcal{I}_{C}(n)$ for $n \geq 4$, since $f^{\infty}(n)=0$ for $n \geq 4$ and $h^{1} \mathcal{I}_{\Gamma}(n)=0$ for $n \geq 4$.

Second we claim that $h^{1} \mathcal{I}_{C}(2)=1$ and $h^{1} \mathcal{I}_{C}(1)=2$. The former is because $f^{\infty}(3)=1$ and $h^{1} \mathcal{I}_{C}(3)=0$. The latter is because $f^{\infty}(2)=1, h^{1} \mathcal{I}_{C}(2)=1$, and $h^{1} \mathcal{I}_{\Gamma}(2)=0$.

Now $F_{2}^{\infty} \subseteq H^{1} \mathcal{I}_{C}(1)$ is the subspace killed by a general linear form $h$. The subspace $F_{2}^{1}$ of $F_{2}^{\infty}$ is the subspace killed by another general linear form $h^{\prime}$. Since $F_{2}^{1}=F_{2}^{\infty}$, this subspace is killed by all linear forms in $S$. The Hartshorne-Rao module therefore decomposes as

$$
H_{*}^{1} \mathcal{I}_{C}=F_{2}^{\infty} \oplus R^{\prime}
$$

where $F_{2}^{\infty}=k[-1]$ and $R^{\prime}$ is a suitable complementary submodule. But computing a minimal curve in the even liaison class from such a Hartshorne-Rao module by the procedure of [11, we see that a minimal curve $D$ will have $H^{1} \mathcal{I}_{D}(n)=0$ for $n \leq 1$. Thus 3 ) cannot be the initial ideal of a rational quintic curve. 
Remark 3.9. Case 3) may be generalized to the fact that if there is an $n$ such that $\tilde{f}^{j}(n-i)=0$ for $j>i>0$, then $F_{n}^{1}=F_{n}^{\infty}$ is a subspace of $H_{*}^{1} \mathcal{I}_{C}(n-1)$ annihilated by all linear forms.

\section{Applichtion to Zero-dimensional schemes}

4.1. In this section we consider a zero-dimensional subscheme $X \subseteq \mathbf{P}^{3}$ and let $I_{X} \subseteq S=k\left[x_{0}, x_{1}, x_{2}, x_{3}\right]$ be its homogeneous ideal. Again we assume that $X$ is in general coordinates, so $\operatorname{in}\left(I_{X}\right)=\operatorname{gin}\left(I_{X}\right)$, and we will seek to understand what information this generic initial ideal contains.

Proposition 4.2. Let $J=I_{X}{ }^{0}$.

a. The initial ideals in $\left(I_{X}\right)$ and in $(J)$ are computationally equivalent.

b. in $(J)$ is computationally equivalent to the Hilbert functions of the modules in the filtration

$$
0=F^{0}(J) \subseteq F^{1}(J) \subseteq \cdots \subseteq F^{\infty}(J)
$$

Proof. This is quite analogous to the proof of Propostion 3.2 when we note the following. Since $X \cap\left\{x_{3}=0\right\}=\emptyset$ we get $\left(J: x_{2}^{\infty}\right)=J^{\text {sat }}=k\left[x_{0}, x_{1}, x_{2}\right]$. Hence we get $J^{\infty}=k\left[x_{0}, x_{1}\right]$.

Remark 4.3. The procedures by which $\operatorname{in}(J)$ and $\operatorname{Hilb}\left(F^{j}(I)\right)$ may be calculated from each other are given by Proposition 2.10 and Algorithm 2.3.

Again we seek to find a more algebraic geometric description of the modules $F^{j}(I)$. This is part a. in the lemma below. Part b. of the lemma below will be used in Section 5.

Lemma 4.4. Let $J=I_{X}^{0}$.

$a$.

$$
F^{\infty}(J)=\operatorname{ker}\left(H_{*}^{1} \mathcal{I}_{X}(-1) \stackrel{\cdot x_{3}}{\longrightarrow} H_{*}^{1} \mathcal{I}_{X}\right) .
$$

$b$.

$$
h^{1} \mathcal{I}_{X}(n)=\sum_{d>n} \operatorname{dim}_{k}\left(k\left[x_{0}, x_{1}, x_{2}\right] / J\right)_{d} .
$$

Proof. There is a short exact sequence of sheaves

$$
0 \longrightarrow \mathcal{I}_{X}(-1) \stackrel{\cdot x_{3}}{\longrightarrow} \mathcal{I}_{X} \longrightarrow \mathcal{O}_{\mathbf{P}^{2}} \longrightarrow 0
$$

giving the long exact sequence

$$
\begin{aligned}
0 & \longrightarrow I_{X}(-1) \stackrel{\cdot x_{3}}{\longrightarrow} I_{X} \stackrel{\alpha}{\longrightarrow} k\left[x_{0}, x_{1}, x_{2}\right] \\
& \longrightarrow H_{*}^{1} \mathcal{I}_{X}(-1) \stackrel{\cdot x_{3}}{\longrightarrow} H_{*}^{1} \mathcal{I}_{X} \longrightarrow H_{*}^{1} \mathcal{O}_{\mathbf{P}^{2}} .
\end{aligned}
$$

We have $\alpha\left(I_{X}\right)=J$ and $\left(J: x_{2}^{\infty}\right)=k\left[x_{0}, x_{1}, x_{2}\right]$, so

$$
F^{\infty}(J)=k\left[x_{0}, x_{1}, x_{2}\right] / J=\operatorname{ker}\left(H_{*}^{1} \mathcal{I}_{X}(-1) \stackrel{\cdot x_{3}}{\longrightarrow} H_{*}^{1} \mathcal{I}_{X}\right) .
$$

Since $H_{*}^{1} \mathcal{O}_{\mathbf{P}^{2}}=0$, we get an exact sequence

$$
0 \rightarrow\left(k\left[x_{0}, x_{1}, x_{2}\right] / J\right)_{d} \rightarrow H^{1} \mathcal{I}_{X}(d-1) \rightarrow H^{1} \mathcal{I}_{X}(d) \rightarrow 0 .
$$

This gives $b$., since $H^{1} \mathcal{I}_{X}(d)=0$ for $d \gg 0$. 
We now get the following.

Theorem 4.5. Let $X \subseteq \mathbf{P}^{3}$ be a zero-dimensional subscheme. Let $h$ and $h^{\prime}$ be general linear forms. Let

$$
F^{\infty}(1)=\operatorname{ker}\left(H_{*}^{1} \mathcal{I}_{X} \stackrel{\cdot h}{\longrightarrow} H_{*}^{1} \mathcal{I}_{X}(1)\right)
$$

and let

$$
F^{j}=\operatorname{ker}\left(F^{\infty} \stackrel{\cdot\left(h^{\prime}\right)^{j}}{\longrightarrow} F^{\infty}(j)\right) .
$$

Then in $\left(I_{X}\right)$ is computationally equivalent to the Hilbert functions of the modules in the filtration

$$
0=F^{0} \subseteq F^{1} \subseteq \cdots \subseteq F^{\infty} .
$$

Proof. Since $\mathcal{I}_{X}$ is in general coordinates, we can consider $x_{2}$ and $x_{3}$ as general linear forms $h^{\prime}$ and $h$. The theorem then follows by Proposition 4.2 and Lemma $4.4 \mathrm{a}$.

\section{Applications to a CURVe intersected With a surface}

5.1. Consider again a space curve $C \subseteq \mathbf{P}^{3}$ with a homogeneous ideal $I_{C} \subseteq S=$ $k\left[x_{0}, x_{1}, x_{2}, x_{3}\right]$. Let $F \subseteq \mathbf{P}^{3}$ be a surface defined by a homogeneous form $f$ of degree $m$. We let $I=I_{C}+(f)$ and will seek to understand what information its generic initial ideal contains. We assume that everything is given in general coordinates so this is just its initial ideal.

Reminder 5.2. From Section 2 we have the following.

1. The first approximation to in $(I)$ is Hilb $\left(I^{j}\right)$ for $j \geq 0$. This is by Remark [2.9.

2. Hilb( $\left.I^{j}\right)$ for $j \geq 0$ is computationally equivalent to:

i. The Hilbert function of $I^{\infty}$.

ii. The Hilbert functions of the modules in the filtration

$$
0=F^{0}(I) \subseteq \cdots \subseteq F^{\infty}(I) .
$$

This is by Algorithm 2.3

3. The procedure to compute the Hilbert functions in 2.i and 2.ii from in $(I)$ is given by Proposition 2.10 and Algorithm 2.3

We now wish to find a more algebraic geometric description of the modules in 2.i. and 2.ii.

The filtration and their Hilbert functions.

\section{Lemma 5.3.}

$$
F^{\infty}(I)=\operatorname{ker}\left(H_{*}^{1} \mathcal{I}_{C}(-m) \stackrel{\cdot f}{\longrightarrow} H_{*}^{1} \mathcal{I}_{C}\right) .
$$

Proof. Let $J$ be the image of the composition $I \longrightarrow S \longrightarrow S /(f)$. From the exact sequences

$$
\begin{aligned}
& 0 \longrightarrow S(-m) \longrightarrow \quad I \longrightarrow \quad J \longrightarrow 0 \text {, } \\
& 0 \longrightarrow S(-m) \longrightarrow I^{\text {sat }} \longrightarrow J^{\text {sat }} \longrightarrow 0
\end{aligned}
$$

we see that $I^{\text {sat }} / I=J^{\text {sat }} / J$. 
There is also a short exact sequence of sheaves on $\mathbf{P}^{3}$ :

$$
0 \longrightarrow \mathcal{I}_{C}(-m) \stackrel{\cdot f}{\longrightarrow} \mathcal{I}_{C} \longrightarrow \mathcal{I}_{C \cap F / F} \longrightarrow 0,
$$

from which we deduce the long exact sequence

$$
\begin{aligned}
0 & \longrightarrow I_{C}(-m) \stackrel{\cdot f}{\longrightarrow} I_{C} \stackrel{\alpha}{\longrightarrow} I_{C \cap F / F} \\
& \longrightarrow H_{*}^{1} \mathcal{I}_{C}(-m) \stackrel{\cdot f}{\longrightarrow} H_{*}^{1} \mathcal{I}_{C} \longrightarrow H_{*}^{1} \mathcal{I}_{C \cap F / F} \\
& \longrightarrow H_{*}^{2} \mathcal{I}_{C}(-m) \stackrel{\cdot f}{\longrightarrow} H_{*}^{2} \mathcal{I}_{C} .
\end{aligned}
$$

Here $I_{C \cap F / F}=J^{\text {sat }}$ and $\alpha\left(I_{C}\right)=J$, so we get

$$
F^{\infty}(I)=J^{\text {sat }} / J=\operatorname{ker}\left(H_{*}^{1} \mathcal{I}_{C}(-m) \stackrel{\cdot f}{\longrightarrow} H_{*}^{1} \mathcal{I}_{C}\right) .
$$

Corollary 5.4. Suppose $C$ is a connected and reduced curve and $m \geq$ (regularity $\left.I_{C}\right)$ - 2. Then $F^{\infty}(I)(m)=H_{*}^{1} \mathcal{I}_{C}$, the Hartshorne-Rao module of $C$.

Proof. Since $C$ is connected and reduced, we have $H^{1} \mathcal{I}_{C}(n)=0$ for $n \leq 0$. Furthermore $H^{1} \mathcal{I}_{C}(n)=0$ for $n \geq$ (regularity $\left.I_{C}\right)-1$. Then if $H^{1} \mathcal{I}_{C}(n) \neq 0$ we must have $1 \leq n \leq$ (regularity $\left.I_{C}\right)-2$. But then if $m=\operatorname{deg} f \geq\left(\right.$ regularity $\left.I_{C}\right)-2$ the multiplication $H_{*}^{1} \mathcal{I}_{C}(-m) \stackrel{\cdot f}{\longrightarrow} H_{*}^{1} \mathcal{I}_{C}$ is zero. Thus we conclude by Lemma 5.2

We now have the following.

Theorem 5.5. Suppose $C$ is a connected and reduced curve and $m \geq$ (regularity $\left.I_{C}\right)-2$. Let $h$ be a general linear form and let

$$
F^{j}(m)=\operatorname{ker}\left(H_{*}^{1} \mathcal{I}_{C} \stackrel{h^{j}}{\longrightarrow} H_{*}^{1} \mathcal{I}_{C}(j)\right) .
$$

Then the Hilbert function of the modules in the filtration

$$
0=F^{0} \subseteq F^{1} \subseteq \cdots \subseteq F^{\infty}
$$

may be computed from in $(I)$.

Proof. Corollary 5.4 gives that $F^{\infty}(I)(m)=H_{*}^{1} \mathcal{I}_{C}$. Since $I$ is in general coordinates,

$$
F^{j}(I)(m)=\operatorname{ker}\left(H_{*}^{1} \mathcal{I}_{C} \stackrel{\cdot x_{3}^{j}}{\longrightarrow} H_{*}^{1} \mathcal{I}_{C}(j)\right)
$$

and $F^{j}(m)$ have the same Hilbert functions. By 1. and 2. in 5.2 it follows that the $\operatorname{Hilb}\left(F^{j}(m)\right)$ can be computed from in $(I)$.

The Hilbert function of $I^{\infty}$. We now investigate what information we may get from $\operatorname{Hilb}\left(I^{\infty}\right)$, which is the data of 2. ii in 5.2 .

Theorem 5.6. Suppose $n \geq\left(\right.$ regularity $\left.I_{C}\right)-m-1$. Then

$$
\begin{aligned}
h^{2} \mathcal{I}_{C}(n) & =\sum_{d>n+m} \operatorname{dim}_{k}\left(k\left[x_{0}, x_{1}, x_{2}\right] / I^{\infty}\right)_{d} \\
& =\#\left\{x^{K}=x_{0}^{k_{0}} x_{1}^{k_{1}} x_{2}^{k_{2}} \mid \operatorname{deg} x^{K}>n+m, x^{K} \notin i n\left(I^{\infty}\right)\right\} .
\end{aligned}
$$


Proof. Let $X=C \cap F$. Then $I_{X}=I^{\text {sat }}=\left(I: x_{3}^{\infty}\right)$ and so $I_{X}{ }^{0}=I^{\infty}$. From Lemma $4.4 \mathrm{~b}$. it follows that

$$
h^{1} \mathcal{I}_{X}(n)=\sum_{d>n} \operatorname{dim}_{k}\left(k\left[x_{0}, x_{1}, x_{2}\right] / I^{\infty}\right)_{d} .
$$

Now there is an exact sequence

$$
0 \rightarrow \mathcal{O}_{\mathbf{P}^{3}}(-m) \rightarrow \mathcal{I}_{X} \rightarrow \mathcal{I}_{C \cap F / F} \rightarrow 0 .
$$

Taking the long exact cohomology sequence, we get

$$
H_{*}^{1} \mathcal{I}_{X}=H_{*}^{1} \mathcal{I}_{C \cap F / F} .
$$

On the other side it follows by sequence (5.1) that if $n \geq$ (regularity $\left.I_{C}\right)-m-1$, then $H^{1} \mathcal{I}_{C \cap F / F}(n+m)=H^{2} \mathcal{I}_{C}(n)$. Together with (5.4) we then get (5.2). The equality (5.3) then follows from (5.2), since $\operatorname{Hilb}\left(I^{\infty}\right)=H i l b\left(\operatorname{in}\left(I^{\infty}\right)\right)$.

Remark 5.7. The interesting consequence of Theorems 5.5 and 5.6 is that we can compute all the cohomological dimensions $h^{i} \mathcal{I}_{C}(n)$ for $i \geq 0$ and $n \in \mathbf{Z}$ from in $(I)$. From the exact sequence

$$
0 \longrightarrow \mathcal{I}_{C}(n) \longrightarrow \mathcal{O}_{\mathbf{P}^{3}}(n) \longrightarrow \mathcal{O}_{C}(n) \longrightarrow 0
$$

the Hilbert polynomial satisfies

$$
\chi \mathcal{I}_{C}(n)=\chi \mathcal{O}_{\mathbf{P}^{3}}(n)-\chi \mathcal{O}_{C}(n),
$$

which becomes (letting $d$ and $g$ be the degree and genus of $C$ )

$$
h^{0} \mathcal{I}_{C}(n)-h^{1} \mathcal{I}_{C}(n)+h^{2} \mathcal{I}_{C}(n)-h^{3} \mathcal{I}_{C}(n)=\left(\begin{array}{c}
n+3 \\
3
\end{array}\right)-(d n+1-g) .
$$

Here $h^{3} \mathcal{I}_{C}(n)=h^{3} \mathcal{O}_{\mathbf{P}^{3}}(n)$. Then, since $h^{1} \mathcal{I}_{C}(n)$ and $h^{2} \mathcal{I}_{C}(n)$ may be computed from in $(I)$, we may also compute $h^{0} \mathcal{I}_{C}(n)$.

Example 5.8. a. Let $C$ be a smooth rational curve of degree 7 with a 6 -secant line, and denote by $I_{C} \subseteq k[x, y, z, w]$ its saturated homogeneous ideal. We assume it to be in general coordinates. We compute on Macaulay that

$$
\operatorname{in}\left(I_{C}\right)=\left(x^{4}, x^{3} y, x^{2} y^{2}, x y^{3}, y^{4}, x^{3} z, x^{2} y z, x y^{2} z^{3}\right) .
$$

The degree of the highest degree generator is here 6 , so $\operatorname{reg}\left(I_{C}\right)=6$. Let $f$ be a general form of degree 8 and let $I=I_{C}+(f)$. Computing in $(I)$, we find the following generators in addition to those listed above:

$$
\begin{array}{lll}
z^{11}, z^{10} w, z^{9} w^{4}, & \\
x z^{9}, x z^{8} w^{2}, & y z^{9}, y z^{8} w^{3}, & \\
x^{2} z^{7}, & x y z^{7}, & y^{2} z^{8}, y^{2} z^{7} w^{2}, \\
y^{3} z^{5} . &
\end{array}
$$

In order to compute the Hilbert functions of the filtration of $F^{\infty}(I)=H_{*}^{1} \mathcal{I}_{C}(-m)$ we compute the $\operatorname{in}\left(I^{j}\right)=\left(\operatorname{in}(I): w^{j}\right)_{\mid w=0}$. We may note that $\operatorname{in}\left(I^{j}\right)=\operatorname{in}\left(I^{\infty}\right)$ for $j \geq 4$. 
For $j=0$ and 1 we get (displaying the function $p$ of the ideal $\operatorname{in}\left(I^{j}\right)$ )
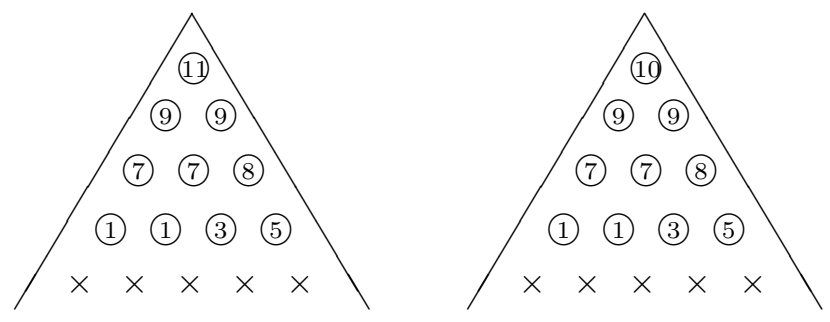

The Hilbert function of $\operatorname{in}\left(I^{1}\right) / \operatorname{in}\left(I^{0}\right)$ is $\tilde{f}^{1}(n)$. We see from the diagrams that $\tilde{f}^{1}(n)=0$ for $n \neq 10$ and $\tilde{f}^{1}(10)=1$. For $j=2,3$ and 4 we get the diagrams for $\operatorname{in}\left(I^{j}\right)$ :
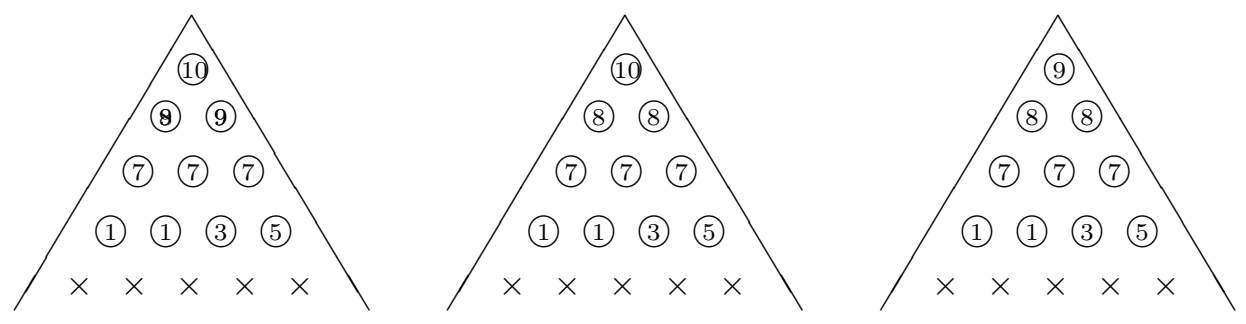

This gives the following table to the left below for the $\tilde{f}^{j}(n)$, which are the Hilbert functions of in $\left(I^{j}\right) / \operatorname{in}\left(I^{j-1}\right)$. By the formula in Algorithm 2.3 we also get the table for the $\bar{f}^{j}(n)$ below to the right.

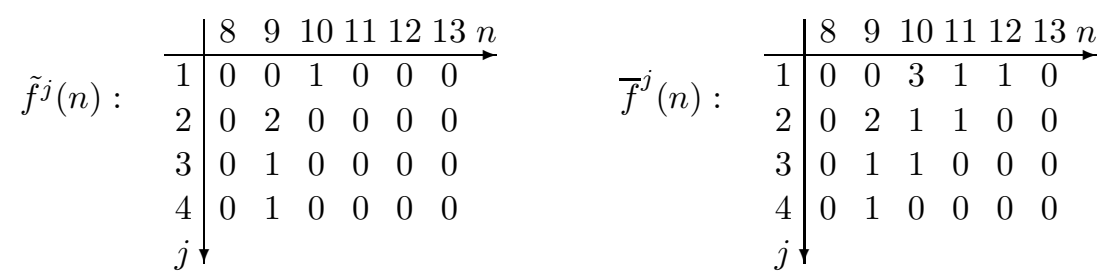

Again by the formula in Algorithm 2.3 we get the table for $f^{j}(n)$ below to the left. Since $H_{*}^{1} \mathcal{I}_{C}=F^{\infty}(I)(m)$ with $m=8$, the Hilbert functions of the filtration of the Hartshorne-Rao module in Theorem [5.5 is given by the table below to the right.

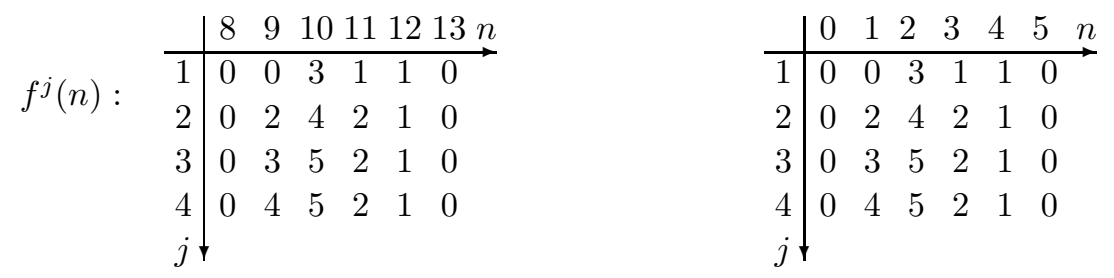


In particular, the last row gives the Hilbert function of the Rao module.

b. The diagram for $j=4$ gives the diagram of in $\left(I^{\infty}\right)$ From this we may, by Theorem 5.6, compute $h^{2} \mathcal{I}_{C}(n)$ for $n \geq$ regularity $I_{C}-m-1=-3$. We find that

\begin{tabular}{r|l|l|l|l|l}
$n$ & -3 & -2 & -1 & 0 & \\
$h^{2} \mathcal{I}_{C}(n)$ & 20 & 13 & 6 & 0 &
\end{tabular}

consistent with the fact that $C$ is a rational curve.

\section{INITIAL MODULES}

The success of investigating the generic initial ideals of curves in $\mathbf{P}^{3}$ suggests that one might also try to understand what information is encoded in the generic higher initial ideal of a curve in $\mathbf{P}^{3}$ (as defined in [5]). In order to do so it will however be necessary to extend most of the theory of Sections 1 and 2 to the case of submodules of a module which does not have a $\mathbf{Z}^{r+1}$-grading. The cleanest approach for what we are going to do here seems to be to axiomatize the concept of an initial module.

6.1. Consider the graded homogeneous ring $S=k\left[x_{0}, \ldots, x_{r}\right]$ and a homogeneous finitely generated module $U$ over $S$. Now $S$ also has a natural $\mathbf{Z}^{r+1}$-grading by letting a monomial

$$
x^{K}=x_{0}^{k_{0}} \cdots x_{r}^{k_{r}}
$$

have degree $K=\left(k_{0}, \ldots, k_{r}\right)$. Let $R$ be a finitely generated module over $S$ homogeneous for this grading. We denote by $R_{K}$ the homogeneous part of $R$ of degree $K$.

Order on $\mathbf{Z}^{r+1}$. Suppose we have also given a total order on $\mathbf{Z}^{r+1}$ satisfying the usual properties of a monomial order, namely, if $I, J$, and $K$ are in $\mathbf{Z}^{r+1}$ and $I>J$, then $I+K>J+K$. If $x$ and $y$ in $Z$ have degrees $I$ and $J$, then write $x>y$ if $I>J$, and write $x \sim y$ if $I=J$.

Definition 6.2. a. An initial map

$$
\text { in }: U \longrightarrow R
$$

is a map (in general not a homomorphism) satisfying the following properties:

i. The map is $\mathbf{Z}$-degree preserving.

ii. If $u \in U, x^{K}$ is a monomial, and $\alpha$ a scalar, then $\operatorname{in}\left(\alpha x^{K} u\right)=\alpha x^{K} \operatorname{in}(u)$.

iii. If in $(u)>\operatorname{in}(v)$, then in $(u+v)=\operatorname{in}(u)$.

iv. Suppose in $(u) \sim \operatorname{in}(v)$. If in $(u)+\operatorname{in}(v) \neq 0$, then $\operatorname{in}(u+v)=\operatorname{in}(u)+\operatorname{in}(v)$. Otherwise in $(u+v)<\operatorname{in}(u)$.

v. If in $(u)=0$, then $u=0$.

The reader may be puzzled by ii., since it is not true the way we defined the initial map for homogeneous ideals in $S$. However, since we cannot assume there to be a canonical element in $R_{K}$, we must have this alternative definition.

b. For a submodule $M \subseteq U$, define

$$
\operatorname{in}(M)=(\{\operatorname{in}(m) \mid m \in M\}),
$$

which is a $\mathbf{Z}^{r+1}$-submodule of $R$. 
Example 6.3. Let $F=\bigoplus S e_{i}$ be a free $S$-module. If we give each $e_{i}$ a $\mathbf{Z}^{r+1}$ grading, then $F$ becomes a $\mathbf{Z}^{r+1}$-graded module over $S$. Then clearly there is an initial map in : $F \rightarrow F$. Suppose we have given submodules $B \subseteq A \subseteq F$. Let $U=A / B$ and let $R=\operatorname{in}(A) / \operatorname{in}(B)$. If $\bar{a}$ is non-zero in $U$, then it is not difficult to verify that there is a lifting $a \in A$ of $\bar{a}$ such that in $(a)$ is not in $\operatorname{in}(B)$. Two such liftings give the same class in $R$. Thus we may define $\operatorname{in}(\bar{a})=\overline{\operatorname{in}(a)}$. That Definition 6.2 i. - v. hold are straightforward to check.

We now give some results for an initial map in $: U \rightarrow R$ and a submodule $M \subseteq U$ which are analogs of results in Sections 1 and 2 concerning the initial map in $: S \rightarrow S$ and an ideal $I \subseteq S$.

The following is the analog of 1.3 .

Proposition 6.4. Let $M$ be a submodule of $U$. Then $M$ and in $(M)$ have the same Hilbert function.

Proof. Given a degree $n \in \mathbf{Z}$, let

$$
K_{1}>\cdots>K_{s}
$$

be the indices of degree $n$ such that $\operatorname{in}(M)_{K_{i}} \neq 0$. We may define a filtration

$$
0 \subseteq F_{s} \subseteq F_{s-1} \subseteq \cdots \subseteq F_{1}=M_{n}
$$

by

$$
F_{i}=\left\{m \in M_{n} \mid \operatorname{in}(m) \leq K_{i}\right\} .
$$

Clearly each $F_{i}$ is a linear subspace of $M_{n}$ by 6.2 ii. - iv. Now $\operatorname{dim}_{k} F_{i} / F_{i+1}=$ $\operatorname{dim}_{k} \operatorname{in}(M)_{K_{i}}$ follows easily by 6.2 i. - v.

6.5. From now on we assume that the following holds:

1. The order on $\mathbf{Z}^{r+1}$ is the reverse lexicographic order.

2. There is a $\mathbf{Z}^{r}$-graded module $R_{\mathbf{l}}$ over $k\left[x_{0}, \ldots, x_{r-1}\right]$ such that

$$
R=R_{\mathbf{1}} \otimes_{k} k\left[x_{r}\right] .
$$

3. The initial map in $: U \rightarrow R$ is surjective.

Lemma 6.6. Let $u \in U$ be such that in $(u)=x_{r} \cdot z$ for some $z \in R$. Then $u=x_{r} \cdot v$ for some $v \in U$.

Proof. Lift $z \in R$ to a $u_{1} \in U$. Then $\operatorname{in}\left(x_{r} \cdot u_{1}\right)=\operatorname{in}(u)$, so

$$
\operatorname{in}\left(u-x_{r} u_{1}\right)<\operatorname{in}(u)=x_{r} z .
$$

By the properties of the revlex order and 2. in 6.5 this gives

$$
\operatorname{in}\left(u-x_{r} u_{1}\right)=x_{r} z_{2}
$$

for a $z_{2}$. There is a $u_{2}$ in $U$ such that $\operatorname{in}\left(u_{2}\right)=z_{2}$. Then

$$
\operatorname{in}\left(u-x_{r} u_{1}-x_{r} u_{2}\right)<x_{r} z_{2} .
$$

In this way we may continue. Since each $\mathbf{Z}$-graded piece of $R$ is finite dimensional we must eventually have in $\left(u-x_{r} \sum u_{i}\right)=0$. Thus 6.2 $\mathrm{v}$. gives $u=x_{r} \sum u_{i}$.

The following is the analog of (1.3) in 1.7.

Corollary 6.7. Let $M \subseteq U$ be a submodule. Then in $\left(M: x_{r}\right)=\operatorname{in}(M): x_{r}$. 
Proof. Taking Lemma [6.6] into consideration, the proof of this is quite analogous to the proof that in $\left(I: x_{r}\right)=\operatorname{in}(I): x_{r}$ for an ideal $I \subseteq S$ when we have the revlex order.

Notation. For $M \subseteq U$ let

$$
M_{\mid x_{r}=0}=\operatorname{im}\left(M \rightarrow U \rightarrow U /\left(x_{r}\right)\right) .
$$

Correspondingly, for $T \subseteq R$ let

$$
T_{\mid x_{r}=0}=\operatorname{im}\left(T \rightarrow R \rightarrow R /\left(x_{r}\right)\right) .
$$

The following is the analog of (1.2) of 1.7 .

Corollary 6.8. $a$. The composition

$$
U \stackrel{\text { in }}{\longrightarrow} R \stackrel{p}{\longrightarrow} R /\left(x_{r}\right)=R_{\mathbf{I}}
$$

factors through $U / x_{r} U$ giving a commutative diagram

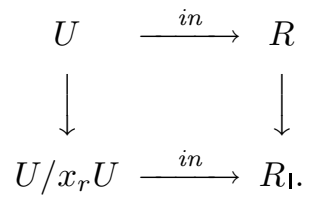

b. The lower horizontal map is an initial map, i.e. it fulfills i. - v. of Definition 6.2.

c. For a submodule $M \subseteq U$ we have

$$
\operatorname{in}\left(M_{\mid x_{r}=0}\right)=i n(M)_{\mid x_{r}=0} .
$$

Proof. a. Let $u, v \in U$ be such that $\bar{u}=\bar{v}$ in $U / x_{r} U$. We wish to show that $p \circ \operatorname{in}(u)=p \circ \operatorname{in}(v)$. We may write $u=v+x_{r} \cdot w$ for a $w \in U$.

a1. If $\operatorname{in}(v)>x_{r} \operatorname{in}(w)$, then $\operatorname{in}(u)=\operatorname{in}(v)$, according to 6.2 iii.

a2. If in $(v)<x_{r} \operatorname{in}(w)$ or in $(v) \sim x_{r} \operatorname{in}(w)$, then in $(v)=x_{r} m$ for an $m \in R$ according to 6.5.1. and 2., and so $u=x_{r} u_{1}$ by Lemma 6.6. Thus $p \circ \operatorname{in}(u)=$ $p \circ \operatorname{in}(v)=0$.

b. Property v. of Definition 6.2 follows by Lemma 6.6. The properties i. - iv. are easy to check.

c. This follows from a. and b.

We now wish to give properties which are analogous to those in Section 2.

6.9. Since $U$ is a Noetherian module, the ascending chain

$$
M \subseteq \cdots \subseteq\left(M: x_{r}^{j-1}\right) \subseteq\left(M: x_{r}^{j}\right) \subseteq \cdots
$$

stabilizes in a module which we denote by $\left(M: x_{r}^{\infty}\right)$. For $j \geq 0$ or $j=\infty$ let

$$
F^{j}(M)=\left(M: x_{r}^{j}\right) / M \text {. }
$$

Notice that for $j \geq 0$ then

$$
F^{j}(M)=\operatorname{ker}\left(U / M \stackrel{x_{r}^{j}}{\longrightarrow} U / M(j)\right) .
$$

Furthermore, for $j \geq 0$ or $j=\infty$ let

$$
M^{j}=\operatorname{im}\left(\left(M: x_{r}^{j}\right) \rightarrow U \rightarrow U /\left(x_{r}\right)\right) .
$$


In quite an analogous way we define for a submodule $T \subseteq R$ submodules $T^{j} \subseteq R_{\mathbf{I}}$ for $j \geq 0$ or $j=\infty$.

The following is an analog of part of Algorithm 2.3

Proposition 6.10. The Hilbert functions of the modules in the filtration

$$
0=F^{0}(M) \subseteq F^{1}(M) \subseteq \cdots \subseteq F^{\infty}(M)
$$

are computationally equivalent to the Hilbert functions of the modules $M^{j} / M^{j-1}$ for $j \geq 0$.

Proof. This is analogous to the proof of Algorithm 2.3.

The following is the analog of Lemma 2.6

Lemma 6.11. Let $T \subseteq R$ be a $\mathbf{Z}^{r+1}$-graded submodule. Then $T$ is computationally equivalent to $T^{j}$ for all $j \geq 0$. In fact, for $z \in R$ we have

$$
z x_{r}^{j} \in T \Leftrightarrow z \in T^{j}
$$

Proof. This is clear by 6.5 , property 2 .

The following is the analog of Lemma 2.7

Lemma 6.12. Let $M \subseteq U$ be a homogeneous submodule. Then in $\left(M^{j}\right)=i n(M)^{j}$.

Proof. We use the commutative diagram from Corollary 6.8 a. on $\left(M: x_{r}^{j}\right) \subseteq U$, noting that by Corollary 6.7 we have $\operatorname{in}\left(M: x_{r}^{j}\right)=\operatorname{in}(M): x_{r}^{j}$.

The following is the analog of Proposition 2.8

Proposition 6.13. Given a submodule $M \subseteq U$ and an initial map in : $U \rightarrow R$ (so that 6.5 1.-3. holds), consider the following data:

i. in $(M), \quad$ ii. $i n\left(M^{j}\right)$ for $j \geq 0$, iii. $\operatorname{Hilb}\left(M^{j}\right)$ for $j \geq 0$.

Then data $i$. and ii. are computationally equivalent, and data iii. can be computed from data ii.

Proof. That data i. and ii. are equivalent is by Lemmata 6.11 and 6.12. That data iii. may be determined by data ii. is Proposition 6.4.

\section{Applications to space CuRVes II}

We consider a space curve $C \subseteq \mathbf{P}^{3}$. Let $I_{C}$ be the saturated homogeneous ideal of $C$. We assume that $I_{C}$ is given in general coordinates. In [5] we established that alongside the generic initial ideal of $I_{C}$, there is also of equal status a generic higher initial ideal of $I_{C}$. With the accomplishment in describing the generic initial ideal of $I_{C}$ in Section 3 let us see if we can get an understanding of the generic higher initial ideal of $I_{C}$. But first we must recall the definition and some basic properties of the higher initial ideal $\operatorname{in}_{1}\left(I_{C}\right)$. Subsections $7.1-7.4$ are preparations before we recall the definition in Subsection 7.5. 
7.1. Recall from Section 3 that $\Gamma=C \cap\left\{x_{3}=0\right\}$ and that $I_{\Gamma}$ is its saturated ideal. By Proposition $2.8 \mathrm{~b}$, $\operatorname{in}\left(I_{\Gamma}\right)$ and $\operatorname{in}\left(I_{\Gamma}^{0}\right)$ are computationally equivalent. Let

$$
L_{-}^{2}\left(I_{C}\right)=k\left[x_{0}, x_{1}\right] / \operatorname{in}\left(I_{\Gamma}^{0}\right) .
$$

This module has a natural $\mathbf{Z}^{2}$-grading, and it is a finite dimensional vector space over the field $k$. Note that this module is computationally equivalent to $\operatorname{in}\left(I_{\Gamma}\right)$, which is computationally equivalent to the numerical character of $C$.

Now let

$$
R_{-}^{2}\left(I_{C}\right)=L_{-}^{2}\left(I_{C}\right)^{*}(2),
$$

where $*$ denotes the vector space dual. Then $R_{-}^{2}\left(I_{C}\right)$ is a $\mathbf{Z}^{2}$-graded $k\left[x_{0}, x_{1}\right]$ module. Furthermore, let

$$
R_{1}^{2}\left(I_{C}\right)=R_{-}^{2}\left(I_{C}\right) \otimes_{k} k\left[x_{2}\right], \quad R^{2}\left(I_{C}\right)=R_{-}^{2}\left(I_{C}\right) \otimes_{k} k\left[x_{2}, x_{3}\right] .
$$

Example 7.2. Consider the curve $C$ given first in the example in Section 3. From the diagram of $I_{\Gamma}{ }^{0}$ we see that $L_{-}^{2}\left(I_{C}\right)$ has a basis $\left(1, x_{0}, x_{1}, x_{0} x_{1}, x_{1}^{2}\right)$ and that $R_{-}^{2}\left(I_{C}\right)$ has a basis $\left(1^{*}(2), x_{0}^{*}(2), x_{1}^{*}(2),\left(x_{0} x_{1}\right)^{*}(2),\left(x_{1}^{2}\right)^{*}(2)\right)$.

\subsection{The function $q$. Now let}

$$
A=\left\{\left(k_{0}, k_{1}\right) \mid x_{0}^{k_{0}} x_{1}^{k_{1}} \neq 0 \text { in } L_{-}^{2}\left(I_{C}\right)\right\} .
$$

Let $p$ be the function of 1.4 associated to the ideal $J=I_{C}{ }^{0}$. Then $A$ is the set of all $\left(k_{0}, k_{1}\right)$ in $\mathbf{N}_{0}^{2}$ such that $p\left(k_{0}, k_{1}\right)=\infty$ by Proposition 2.10.

Let $T_{\mathbf{l}}^{2} \subseteq R_{\mathbf{l}}^{2}\left(I_{C}\right)$ be a $\mathbf{Z}^{3}$-homogeneous submodule. We define a function $q$ : $A \rightarrow \mathbf{N}_{0} \cup\{\infty\}$ by

$$
q\left(k_{0}, k_{1}\right)=\min \left\{n \mid\left(x_{0}^{i_{0}} x_{1}^{i_{1}}\right)^{*}(2) \cdot x_{2}^{n} \in T_{\mathbf{I}}^{2}\right\} .
$$

Note that if coker $\left(T_{\mathrm{l}}^{2} \rightarrow R_{1}^{2}\left(I_{C}\right)\right)$ has finite length, then $q\left(k_{0}, k_{1}\right)<\infty$ for all $\left(k_{0}, k_{1}\right) \in A$.

The higher initial ideal. We recall the definition of the higher initial ideal $\operatorname{in}_{1}\left(I_{C}\right)$ given in [5], Section 4. We constructed in [5], (4.4), a resolution

$$
F^{-2} \stackrel{d^{-2}}{\longrightarrow} F^{-1} \stackrel{d^{-1}}{\longrightarrow} F^{0} \stackrel{d^{0}}{\longrightarrow} I_{C},
$$

where $F^{-i}$ are free $S$-modules. This resolution has the property that the maps $d^{-i}$ may be deformed to maps $d_{\succ}^{-i}$ such that

$$
F^{-2} \stackrel{d_{\succ}^{-2}}{\longrightarrow} F^{-1} \stackrel{d_{\succ}^{-1}}{\longrightarrow} F^{0} \stackrel{d_{\succ}^{0}}{\longrightarrow} \operatorname{in}\left(I_{C}\right)
$$

is a minimal resolution of the initial ideal. This also gives each module $F^{-i}$ a natural $\mathbf{Z}^{4}$-grading. If we dualize these complexes, they become

$$
\begin{aligned}
& 0 \rightarrow S \stackrel{d^{0 \vee}}{\longrightarrow} F^{0 \vee} \stackrel{d^{-1} \vee}{\longrightarrow} F^{-1 \vee} \stackrel{d^{-2 \vee}}{\longrightarrow} F^{-2 \vee}, \\
& 0 \rightarrow S \stackrel{d_{\succ}^{0 \vee}}{\longrightarrow} F^{0 \vee} \stackrel{d_{\succ}^{-1 \vee}}{\longrightarrow} F^{-1 \vee} \stackrel{d_{\succ}^{-2 \vee}}{\longrightarrow} F^{-2 \vee} .
\end{aligned}
$$

Note that these complexes are exact in the components of degree -1 and 0 . At the components of degree 1 we have the following. 
Fact 7.4. Let

$$
\begin{array}{rlrl}
Z^{2}\left(I_{C}\right) & =\operatorname{ker} d_{\succ}^{-2 \vee}, & & B^{2}\left(I_{C}\right)=\operatorname{im} d_{\succ}^{-1 \vee}, \\
K^{2}\left(I_{C}\right)=\operatorname{ker} d^{-2 \vee}, & & D^{2}\left(I_{C}\right)=\operatorname{im} d^{-1 \vee} .
\end{array}
$$

Then the following relations hold:

a. $R^{2}\left(I_{C}\right)=Z^{2}\left(I_{C}\right) / B^{2}\left(I_{C}\right)$.

b. $B^{2}\left(I_{C}\right) \subseteq i n\left(D^{2}\left(I_{C}\right)\right) \subseteq i n\left(K^{2}\left(I_{C}\right)\right) \subseteq Z^{2}\left(I_{C}\right)$.

c. $\operatorname{in}\left(D^{2}\left(I_{C}\right)\right)=B^{2}\left(I_{C}\right)$.

Proof. a. This is by [5], Proposition 1.9 and Subsection 4.6.

b. The inclusions $B^{2}\left(I_{C}\right) \subseteq \operatorname{in}\left(K^{2}\left(I_{C}\right)\right) \subseteq Z^{2}\left(I_{C}\right)$ are by [5], Lemma 2.11 (see also Subsection 4.6). Also, $\operatorname{in}\left(D^{2}\left(I_{C}\right)\right) \subseteq \operatorname{in}\left(K^{2}\left(I_{C}\right)\right)$ because $D^{2}\left(I_{C}\right) \subseteq K^{2}\left(I_{C}\right)$, and $B^{2}\left(I_{C}\right) \subseteq \operatorname{in}\left(D^{2}\left(I_{C}\right)\right)$ follows by the proof of [5], Lemma 2.11 .

c. There are two short exact sequences

$$
\begin{aligned}
& 0 \longrightarrow S \stackrel{d^{0 \vee}}{\longrightarrow} F^{0} \stackrel{d^{-1 \vee}}{\longrightarrow} D^{2}\left(I_{C}\right) \longrightarrow 0, \\
& 0 \longrightarrow S \stackrel{d_{\succ}^{0 \vee}}{\longrightarrow} F^{0} \stackrel{d_{\succ}^{-1 \vee}}{\longrightarrow} B^{2}\left(I_{C}\right) \longrightarrow 0 .
\end{aligned}
$$

Hence $B^{2}\left(I_{C}\right)$ and $\operatorname{in}\left(D^{2}\left(I_{C}\right)\right)$ have the same Hilbert functions. By the inclusion in $b$. we must then have the equality in $c$.

Definition 7.5. The higher initial ideal $\operatorname{in}_{1}\left(I_{C}\right)$ is the inclusion

$$
T^{2}\left(I_{C}\right)=\operatorname{in}\left(K^{2}\left(I_{C}\right)\right) / B^{2}\left(I_{C}\right) \subseteq Z^{2}\left(I_{C}\right) / B^{2}\left(I_{C}\right)=R^{2}\left(I_{C}\right) .
$$

Note. Since we are assuming that $I_{C}$ is in general coordinates, this is of course the same as the generic higher initial ideal of $I_{C}$, and our further results for $\operatorname{in}_{1}\left(I_{C}\right)$ are thus results for the generic higher initial ideal of $I_{C}$.

Convention. For the rest of this section we write

$$
Z^{2}=Z^{2}\left(I_{C}\right), \quad K^{2}=K^{2}\left(I_{C}\right), \quad B^{2}=B^{2}\left(I_{C}\right), \quad D^{2}=D^{2}\left(I_{C}\right)
$$

for ease of notation.

We would now be in a position to apply the theory of Section 6 to the initial map

$$
\text { in : } K^{2} / D^{2} \rightarrow \operatorname{in}\left(K^{2}\right) / \operatorname{in}\left(D^{2}\right)=T^{2}\left(I_{C}\right) \subseteq R^{2}\left(I_{C}\right) .
$$

However, in analogy with the case of the initial ideal, we shall rather use an initial map mapping to $R_{1}^{2}\left(I_{C}\right)$. We continue to prepare the ground for this situation.

7.6. Since $\operatorname{in}\left(I_{C}\right)$ is monomial and $\operatorname{in}\left(I_{C}\right): x_{3}=\operatorname{in}\left(I_{C}\right)$, it follows that $\operatorname{in}\left(I_{C}\right)=$ $\operatorname{in}\left(I_{C}{ }^{0}\right) \otimes_{k} k\left[x_{3}\right]$. Now let

$$
F_{1}^{-2} \stackrel{d_{\succ 1}^{-2}}{\longrightarrow} F_{1}^{-1} \stackrel{d_{\succ 1}^{-1}}{\longrightarrow} F_{1}^{0} \longrightarrow \operatorname{in}\left(I_{C}{ }^{0}\right)
$$

be a minimal resolution, where $F_{1}^{-i}=\bigoplus_{j} k\left[x_{0}, x_{1}, x_{2}\right] e_{j}^{-i}$ are free $k\left[x_{0}, x_{1}, x_{2}\right]$ modules. Then each $F_{\mid}^{-i}$ has a $\mathbf{Z}^{3}$-grading.

The minimal resolution of $\operatorname{in}\left(I_{C}\right)$ is then

$$
\left(F^{\bullet}, d\right)=\left(F_{\mathbf{i}}, d_{\succ 1}\right) \otimes_{k} k\left[x_{3}\right] .
$$


By Corollary 6.8 we have a commutative diagram

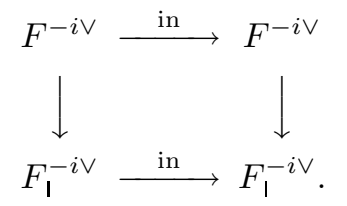

Lemma 7.7. Let $M \subseteq F^{-i \vee}$ be a submodule. Then:

a. $i n(M)_{\mid x_{3}=0}=\operatorname{in}\left(M_{\mid x_{3}=0}\right)$.

b. If $M: x_{3}=M$, then

$$
i n(M)=i n(M)_{\mid x_{3}=0} \otimes_{k} k\left[x_{3}\right]
$$

Proof. a. This is Corollary 6.8 c., applied to the initial map in : $F^{-i \vee} \rightarrow F^{-i \vee}$.

b. If $M=M: x_{3}$, then $\operatorname{in}(M)=\operatorname{in}\left(M: x_{3}\right)=\operatorname{in}(M): x_{3}$, where the last equality is by Corollary 6.7. But then we easily see that

$$
\operatorname{in}(M)=\operatorname{in}(M)_{\mid x_{3}=0} \otimes_{k} k\left[x_{3}\right]
$$

Corollary 7.8. We have:

a. $B^{2}=B_{\mid x_{3}=0}^{2} \otimes_{k} k\left[x_{3}\right]$.

b. $Z^{2}=Z_{\mid x_{3}=0}^{2} \otimes_{k} k\left[x_{3}\right]$.

c. $\operatorname{in}\left(D^{2}\right)=\operatorname{in}\left(D^{2}\right)_{\mid x_{3}=0} \otimes_{k} k\left[x_{3}\right]=i n\left(D_{\mid x_{3}=0}^{2}\right) \otimes_{k} k\left[x_{3}\right]$.

d. $i n\left(K^{2}\right)=i n\left(K^{2}\right)_{\mid x_{3}=0} \otimes_{k} k\left[x_{3}\right]=i n\left(K_{\mid x_{3}=0}^{2}\right) \otimes_{k} k\left[x_{3}\right]$.

Proof. a. and b. follow since the resolution $\left(F^{\bullet}, d_{\succ}\right)$ of $\operatorname{in}\left(I_{C}\right)$ is given by

$$
\left(F^{\bullet}, d_{\succ}\right)=\left(F_{\mathbf{i}}^{\bullet}, d_{\succ}\right) \otimes_{k} k\left[x_{3}\right] .
$$

c. follows by the equality $B^{2}=\operatorname{in}\left(D^{2}\right)$ and Lemma [7.7 a.

d. follows by Lemma $7.7 \mathrm{a}$. and b.

Fact/Lemma 7.9. Let $T_{\mathbf{I}}^{2}\left(I_{C}\right)=T^{2}\left(I_{C}\right)_{\mid x_{3}=0}$ and let $i$ be the inclusion $T_{\mathbf{I}}^{2}\left(I_{C}\right) \subseteq$ $R_{\mathbf{l}}^{2}\left(I_{C}\right)$.

a. The inclusion $T^{2}\left(I_{C}\right) \subseteq R^{2}\left(I_{C}\right)$ identifies naturally with $i \otimes_{k} k\left[x_{3}\right]$.

b. The cokernel of $i$ has finite length.

Proof. a. This holds by Corollary 7.8 since

$$
T^{2}\left(I_{C}\right)=\operatorname{in}\left(K^{2}\right) / \operatorname{in}\left(D^{2}\right) \subseteq Z^{2} / B^{2}=R^{2}\left(I_{C}\right) .
$$

b. This is [5], Lemma $4.7 \mathrm{~b})$.

The following will make it possible to relate $\operatorname{in}_{1}\left(I_{C}\right)$ (which is $T^{2}\left(I_{C}\right) \subseteq R^{2}\left(I_{C}\right)$ ) to algebraic geometric properties of the curve $C$.

Fact/Lemma 7.10. Let $C^{1}=\operatorname{coker}\left(H_{*}^{1} \mathcal{I}_{C}(-1) \stackrel{\cdot x_{3}}{\longrightarrow} H_{*}^{1} \mathcal{I}_{C}\right)$.

a. There is an exact sequence

$$
0 \longrightarrow K^{2}(-1) \stackrel{\cdot x_{3}}{\longrightarrow} K^{2} \longrightarrow\left(K_{\mid x_{3}=0}^{2}: x_{2}^{\infty}\right) \longrightarrow\left(C^{1}\right)^{*}(3) \longrightarrow 0 .
$$

b. $i n\left(K_{\mid x_{3}=0}^{2}: x_{2}^{\infty}\right)=i n\left(K_{\mid x_{3}=0}^{2}\right): x_{2}^{\infty}$.

c. $\operatorname{in}\left(K_{\mid x_{3}=0}^{2}\right): x_{2}^{\infty}=Z_{\mid x_{3}=0}^{2}$. 
Proof. The complex $\left(F^{\bullet}, d\right)$ from (7.1) restricted to $x_{3}=0$ gives a complex $\left(F_{\mathbf{i}}, d_{\mathbf{l}}\right)$.

We have that $K_{\mid x_{3}=0}^{2} \subseteq \operatorname{ker}\left(F_{\mathbf{I}}^{-1 \vee} \stackrel{d_{1}^{-2 \vee}}{\longrightarrow} F_{\mathbf{I}}^{-2 \vee}\right)$, and since the quotient of this inclusion has finite length we get

$$
K_{\mid x_{3}=0}^{2} s a t=\left(K_{\mid x_{3}=0}^{2}: x_{2}^{\infty}\right)=\operatorname{ker}\left(F_{\mid}^{-1 \vee} \stackrel{d_{1}^{-2 \vee}}{\longrightarrow} F_{1}^{-2 \vee}\right) .
$$

a. This is 5, Proposition 12.7, and (7.3).

b. This follows by the fourth line in the proof of Proposition 12.8 in [5].

c. By Corollary 7.8 and Fact/Lemma 7.9 a. we have a diagram

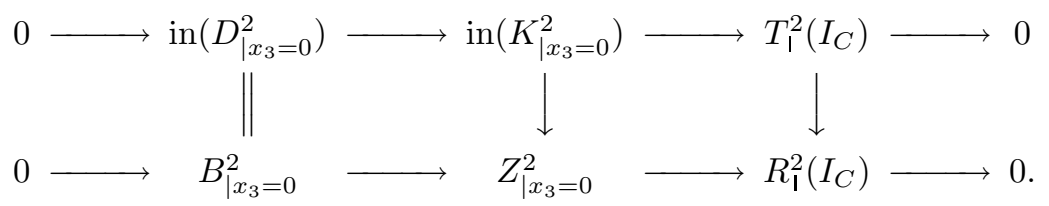

By Fact/Lemma 7.9 b. it then follows that the cokernel of

$$
\operatorname{in}\left(K_{\mid x_{3}=0}^{2}\right) \rightarrow Z_{\mid x_{3}=0}^{2}
$$

has finite length. Since

$$
Z_{\mid x_{3}=0}^{2}=\operatorname{ker}\left(F_{\mathbf{I}}^{-1 \vee} \stackrel{d_{\succ l}^{-2 \vee}}{\longrightarrow} F_{\mid}^{-2 \vee}\right),
$$

we have $\left(Z_{\mid x_{3}=0}^{2}: x_{2}\right)=Z_{\mid x_{3}=0}^{2}$. Thus we get

$$
\operatorname{in}\left(K_{\mid x_{3}=0}^{2}\right): x_{2}^{\infty}=Z_{\mid x_{3}=0}^{2} .
$$

Proposition 7.11. The following hold.

$$
\begin{aligned}
& \text { a. } T_{1}^{2}\left(I_{C}\right)=i n\left(K_{\mid x_{3}=0}^{2}\right) / i n\left(D_{\mid x_{3}=0}^{2}\right) . \\
& \text { b. } R_{\mathbf{1}}^{2}\left(I_{C}\right)=i n\left(K_{\mid x_{3}=0}^{2}: x_{2}^{\infty}\right) / i n\left(D_{\mid x_{3}=0}^{2}\right) .
\end{aligned}
$$

Proof. a. This follows by Fact 7.4k. and Corollary 7.8 .

b. We have $R_{1}^{2}\left(I_{C}\right)=Z_{\mid x_{3}=0}^{2} / B_{\mid x_{3}=0}^{2}$. According to the above Fact/Lemma 7.10 we have $Z_{\mid x_{3}=0}^{2}=\operatorname{in}\left(K_{\mid x_{3}=0}^{2}: x_{2}^{\infty}\right)$, and according to Fact 7.4k. and Corollary 7.8 we have $B_{\mid x_{3}=0}^{2}=\operatorname{in}\left(D_{\mid x_{3}=0}^{2}\right)$.

7.12. We have now come so far that we can apply the theory in Section 6 as indicated by Example 6.3. We let

$$
U=\left(K_{\mid x_{3}=0}^{2}: x_{2}^{\infty}\right) / D_{\mid x_{3}=0}^{2}, \quad M=K_{\mid x_{3}=0}^{2} / D_{\mid x_{3}=0}^{2}, \quad R=R_{\mathbf{1}}^{2}\left(I_{C}\right) .
$$

Proposition 7.13. Let $C \subseteq \mathbf{P}^{3}$ be a space curve.

a. $i n_{1}\left(I_{C}\right)$ is computationally equivalent to $T_{1}^{2}\left(I_{C}\right) \subseteq R_{1}^{2}\left(I_{C}\right)$.

b. $T_{1}^{2}\left(I_{C}\right) \subseteq R_{1}^{2}\left(I_{C}\right)$ is computationally equivalent to $R_{1}^{2}\left(I_{C}\right)$ and

i. the Hilbert function of $M^{\infty}$,

ii. the Hilbert functions of the modules in the filtration

$$
0=F^{0}(M) \subseteq F^{1}(M) \subseteq \cdots \subseteq F^{\infty}(M) .
$$


Proof. a. This is Fact/Lemma 7.9 a.

b. Suppose $R_{1}^{2}\left(I_{C}\right)=R_{-}^{2}\left(I_{C}\right) \otimes_{k} k\left[x_{3}\right]$ is given. By Lemma 6.11, $T_{1}^{2}\left(I_{C}\right)$ is computationally equivalent to $T_{1}^{2}\left(I_{C}\right)^{j}=\operatorname{in}\left(M^{j}\right)$ for $j \geq 0$. Now in $\left(M^{j}\right) \subseteq R_{-}^{2}\left(I_{C}\right)$, and $R_{-}^{2}\left(I_{C}\right)$ is a $\mathbf{Z}^{2}$-graded module.

The order $x_{0}>x_{1}>x_{2}>x_{3}$ gives rise to a natural Borel group $B \subseteq G L\left(S_{1}\right)$ of upper triangular matrices. By [5], (6.5), the group $B$ acts on $R^{2}\left(I_{C}\right)$ and $R_{-}^{2}\left(I_{C}\right)$, and $T^{2}\left(I_{C}\right)$ is invariant under this action ([5], Theorem (8.2)), and hence $T_{1}^{2}\left(I_{C}\right)^{j} \subseteq$ $R_{-}^{2}\left(I_{C}\right)$ is also invariant. It is then easily seen in analogy with the case of a Borel fixed ideal $J \subseteq k\left[x_{0}, x_{1}\right]$ that $T_{1}^{2}\left(I_{C}\right)^{j} \subseteq R_{-}^{2}\left(I_{C}\right)$ is computationally equivalent to the Hilbert function of $T_{1}^{2}\left(I_{C}\right)^{j}$.

Hence in $\left(M^{j}\right)$ for $j \geq 0$ is computationally equivalent to $\operatorname{Hilb}\left(M^{j}\right)$ for $j \geq 0$. By Proposition 6.10 this is computationally equivalent to

i. The Hilbert function of $M^{\infty}$.

ii. The Hilbert functions of the modules in the filtration

$$
0=F^{0}(M) \subseteq \cdots \subseteq F^{\infty}(M) .
$$

We now seek to find a more algebraic geometric description of the modules involved in the above Proposition 7.13 Recall the module $C^{1}$ from Fact/Lemma 7.10.

Lemma 7.14. Given a space curve $C \subseteq \mathbf{P}^{3}$.

a. $F^{\infty}(M)=\left(C^{1}\right)^{*}(3)$.

b. in $\left(M^{\infty}\right)=R_{-}^{2}\left(I_{C}\right)$, and $\operatorname{Hilb}\left(R_{-}^{2}\left(I_{C}\right)\right)$ is computationally equivalent to the numerical character of $C$.

Proof. a. We have $F^{\infty}(M)=\left(M: x_{2}^{\infty}\right) / M$. By Corollary 6.7, Proposition 7.11 a., and Fact/Lemma 7.9 b. we have

$$
\operatorname{in}\left(M: x_{2}^{\infty}\right)=\operatorname{in}(M): x_{2}^{\infty}=T_{1}^{2}\left(I_{C}\right): x_{2}^{\infty}=R_{1}^{2}\left(I_{C}\right) .
$$

Hence

$$
M: x_{2}^{\infty}=U=\left(K_{\mid x_{3}=0}^{2}: x_{2}^{\infty}\right) / D_{\mid x_{3}=0}^{2} .
$$

Thus if follows that

$$
F^{\infty}(M)=\left(K_{\mid x_{3}=0}^{2}: x_{2}^{\infty}\right) / K_{\mid x_{3}=0}^{2}
$$

and by Fact/Lemma 7.10, a. this is equal to $\left(C^{1}\right)^{*}(3)$.

b. Since $\operatorname{in}(M)=T_{1}^{2}\left(I_{C}\right)$, we get

$$
\operatorname{in}\left(M^{\infty}\right)=T_{1}^{2}\left(I_{C}\right)^{\infty}=R_{-}^{2}\left(I_{C}\right) .
$$

By the definition of $R_{-}^{2}$ it is computationally equivalent to in $\left(I_{\Gamma}^{0}\right)$, which by Lemma 3.4 and Remark 3.5 is computationally equivalent to the numerical character.

We now get the main result of this section.

Theorem 7.15. Let $C \subseteq \mathbf{P}^{3}$ be a space curve. Let $h$ and $h^{\prime}$ be general linear forms. Let $\left(F^{\infty}\right)^{*}(3)$ be the cokernel of the map

$$
H_{*}^{1} \mathcal{I}_{C}(-1) \stackrel{\cdot h}{\longrightarrow} H_{*}^{1} \mathcal{I}_{C}
$$


and let $\left(F^{j}\right)^{*}$ be the cokernel of the map

$$
\left(F^{\infty}\right)^{*}(-j) \stackrel{\cdot\left(h^{\prime}\right)^{j}}{\longrightarrow}\left(F^{\infty}\right)^{*}
$$

Then $i n_{1}\left(I_{C}\right)$ is computationally equivalent to:

i. The numerical character of $C$.

ii. The Hilbert function of the successive quotients

$$
\left(F^{\infty}\right)^{*} \rightarrow \cdots \rightarrow\left(F^{2}\right)^{*} \rightarrow\left(F^{1}\right)^{*} \rightarrow\left(F^{0}\right)^{*}=0 .
$$

Proof. Recall that $C^{1}$ is the cokernel of the map

$$
H_{*}^{1} \mathcal{I}_{C}(-1) \stackrel{\cdot x_{3}}{\longrightarrow} H_{*}^{1} \mathcal{I}_{C} .
$$

Since $F^{\infty}(M)=\left(C^{1}\right)^{*}(3)$ by Lemma 7.14 a., we see that $C^{1}=\left(F^{\infty}(M)\right)^{*}(3)$. We may then reach our desired conclusion by Proposition 7.13 and Lemma 7.14 b. modulo the following observation :

Since $I_{C}$ is in general coordinates, we may assume $x_{2}$ and $x_{3}$ to be general linear forms $h^{\prime}$ and $h$ when giving a Hilbert function.

The above theorem has the following interesting consequence.

Corollary 7.16. Suppose a curve $C$ is linked to a curve $X$ by surfaces of given degree $s$ and $t$. Then giving $i n_{1}\left(I_{C}\right)$ is equivalent to giving in $\left(I_{X}\right)$.

Proof. If $C$ is linked to $X$ by surfaces of degree $s$ and $t$, then the numerical character of $C$ is equivalent to giving the numerical character of $X$.

Furthermore, there is the following relation between the Hartshorne-Rao modules by [11], III.1:

$$
H_{*}^{1} \mathcal{I}_{X}=\left(H_{*}^{1} \mathcal{I}_{C}\right)^{*}(-s-t+4) .
$$

This shows that i. and ii. in Theorem 3.7 for the curve $X$ are computationally equivalent to i. and ii. in Theorem 7.15 for the curve $C$.

Example 7.17. Suppose that we have a curve $C$ and link it $(s, t)=(4,5)$, and get a curve $X$ where in $\left(I_{X}\right)$ is given by the following diagram:

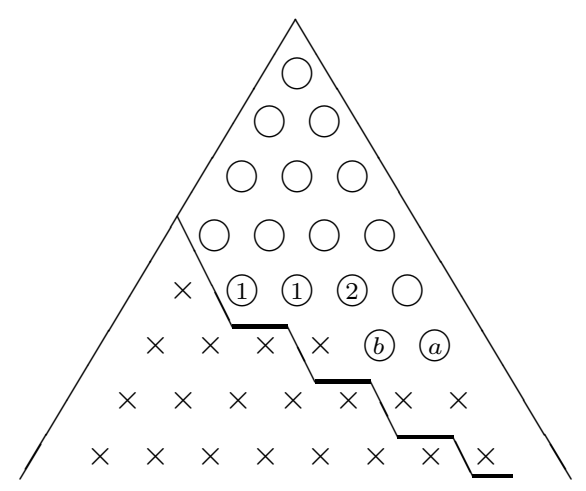

The step line is there to indicate what the initial ideal of a complete intersection $(4,5)$ will be. Since the Rao module $H_{*}^{1} \mathcal{I}_{X} \cong\left(H_{*}^{1} \mathcal{I}_{C}\right)^{*}(-s-t+4)=\left(H_{*}^{1} \mathcal{I}_{C}\right)^{*}(-5)$ 
we get that $\operatorname{in}_{1}\left(I_{C}\right)$ is represented by the following diagram, where we give the values of $q\left(k_{0}, k_{1}\right)$ :

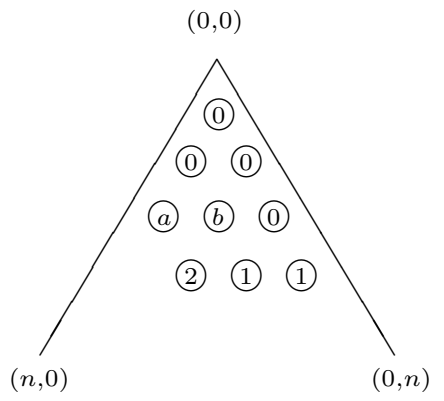

An interesting observation is that by Borel-fixedness of $\operatorname{in}_{1}\left(I_{C}\right)$ ([5], Section 8) we must have $a \leq 1$ and $b=0$ (see [5, (13.9)). This means that if we have a curve $X$ with initial ideal as given above, lying on a complete intersection $(4,5)$ (this holds if $X$ is integral), then we must have $a \leq 1$ and $b=0$.

\section{REFERENCES}

[1] D. Bayer, M. Stillman. A criterion for detecting m-regularity. Inventiones Matematicae $\mathbf{8 7}$ (1987), 1-11. MR 87k:13019

[2] C. v. Bothmer. Thesis, University of Bayreuth (1995).

[3] M. Cook. The connectedness of space curve invariants. Compositio Mathematica 111 no.2 (1998). MR 99a:14040

[4] D. Eisenbud. Commutative algebra with a view towards algebraic geometry. Springer-Verlag, 1995. MR 97a:13001

[5] G. Fløystad. Higher initial ideals of homogeneous ideals. Memoirs of the American Mathematical Society 134 no. 638 (1998). MR 98m:13021

[6] G. Fløystad. A property deducible from the generic initial ideal. Journal of Pure and Applied Algebra 136 (1999), 127-140. MR 2000c:13039

[7] A. Galligo. A propos du theoreme de preparation de Weierstrass. Fonctions de Plusieurs Variables Complexes (Sém. François Norquet, 1970-1972), Lecture Notes in Mathematics, vol. 409, Springer-Verlag, 1973, pp. 543-579. MR 53:5924

[8] M. Green. Generic initial ideals. Summer school on commutative algebra, Barcelona 16. - 26. July 1996. Accompanying notes, volume II.

[9] L. Gruson, C. Peskine. Genre des courbes de l'espace projectif, Algebraic Geometry: Proc. Symposium University of Troms $\varnothing, 1977$, Lecture Notes in Mathematics, vol. 687, SpringerVerlag, 1978, pp. 31-59. MR 81e:14019

[10] R. Liebling. Classification of space curves using initial ideals. Ph.D. thesis, University of California at Berkeley, (1996).

[11] M. Martin-Deschamps, D. Perrin. Sur la classification des courbes gauches. Astérisque 184185 (1990). MR 91h:14039

Matematisk Institutt, Johs. Brunsgate 12, 5008 Bergen, Norway

E-mail address: gunnar@mi.uib.no

Department of Mathematics, University of California at los Angeles, Los Angeles, CALIFORNia 90024

E-mail address: mlg@math.ucla.edu 